\title{
Hopf bifurcation and chaos control for a Leslie-Gower type generalist predator model
}

Qin Chen ${ }^{1}$ and Jianguo Gao ${ }^{1 *}$

\section{"Correspondence:} gaojguo@163.com ${ }^{1}$ School of Mathematics and Information Science, North Minzu University, Yinchuan, China

\section{Springer}

\begin{abstract}
This paper is concerned with chaos control and bifurcations of the Leslie-Gower type generalist predator model in a tri-trophic food web system with the time-delayed feedback control. First, the distribution of the roots of the related characteristic equations is analyzed by the polynomial theorem, the conditions to guarantee the existence of Hopf bifurcation are given by choosing the time delay as a bifurcation parameter. Then, the explicit formula for direction of Hopf bifurcation and stability of periodic solutions bifurcating are determined by using the normal form theory and center manifold theorem. Finally, the correctness of our theoretical analysis is verified by some numerical simulation.
\end{abstract}

Keywords: Hopf bifurcation; Chaos; Delay feedback control

\section{Introduction}

Chaos has attracted considerable attention since the discovery of Lorenz chaotic attractor. After that, many chaotic systems, such as Rössler system [1], Chua circuit [2], and Chen system [3], have been widely studied and applied. Chaos, which often causes irregular behaviors in a practical system, is usually undesirable. Therefore, chaos control is the amusing and challenging topic of our present work [4-11].

The control of chaos involves eliminating and restraining the chaos phenomenon when it is unavailable and harmful. It has been noticed that purposeful control of chaos can be a key issue in many technological applications [12-15]. Generally, the existing chaos control methods can be divided into two categories, feedback control and non-feedback control, according to their characteristics. The most representative methods among chaos control include parameter perturbation control (OGY), delayed feedback control (DFC), occasional proportional feedback (OPF), adaptive control, chaotic signal synchronization control, and other methods. It has been confirmed that the DFC method is exceedingly successful for stabilizing unstable periodic orbits. The DFC method, first introduced by Pyragas, seems more applicable to stabilizing unstable periodic orbits because it can construct a control force from the current state to the periodic state. Since the DFC is an effective method for chaos control, it has become a field of increasing interest and has been applied to some real systems such as biological system, electronic system, communication system, financial system, chemical system, and so on. As an example, Zhao, Lin, and Dai investigated the chaos phenomena of three species food chain models using the method

(c) The Author(s) 2019. This article is distributed under the terms of the Creative Commons Attribution 4.0 International License (http://creativecommons.org/licenses/by/4.0/), which permits unrestricted use, distribution, and reproduction in any medium, provided you give appropriate credit to the original author(s) and the source, provide a link to the Creative Commons license, and indicate if changes were made. 
of delayed feedback control in [16]. Their results show that, when the controlling parameter $\mathrm{K}$ is some value, taking the delay as the bifurcation parameter, then passing through a certain critical value, the stability of the equilibrium will be changed from unstable to stable, chaos will vanish, and a periodic solution will emerge.

The recent findings confirm that systems that appeared in biology also have a chaos phenomenon. In the sense of biology, chaos is a disadvantageous factor in the virtuous cycle and development of ecosystems, so we intend to control this chaos phenomenon by the DFC method. Priyadarshi and Gakkhar [17] proposed a tri-trophic food web model with Leslie-Gower type generalist predator as follows:

$$
\left\{\begin{array}{l}
\frac{d X}{d T}=X g(X)-Y p(X)-Z q_{1}(X, Y), \\
\frac{d Y}{d T}=Y[-r+c p(X)]-Z q_{2}(X, Y), \\
\frac{d Z}{d T}=S_{0}\left(Z^{2}-\frac{Z^{2}}{S_{3}+S_{1} X+S_{2} Y}\right),
\end{array}\right.
$$

where $X$ represents the density of the bottom prey, $Y$ represents the density of the specialist predator, and $Z$ represents the density of the generalist predator. $r$ is the death rate of the specialist predator in absence of the bottom prey, and $c$ is its conversion efficiency. The two predators get food from the bottom prey, while the generalist predator also preys on the specialist predator. The bottom prey is growing logistically, the specialist predator predates on the bottom prey according to Holling type II functional response, and the Leslie-Gower type dynamics is considered for the generalist predator. Therefore $g(X)=a_{0}\left(1-\frac{X}{K}\right), p(X)=\frac{a_{1} X}{1+b_{1} X}, q_{1}(X, Y)=\frac{a_{2} X}{1+b_{2} X+b_{3} Y}, q_{2}(X, Y)=\frac{a_{3} Y}{1+b_{2} X+b_{3} Y}$. Here, $a_{0}$ is the intrinsic growth rate of the bottom prey, $K$ is the carrying capacity of the environment. $a_{2}$ and $a_{3}$ are the maximum grazing rate of the generalist predator with respect to the bottom prey and the specialist predator, respectively. $b_{2}$ and $b_{3}$ represent the coefficients of food taken by the generalist predator from the bottom prey and the specialist predator, respectively. $S_{0}$ is the intrinsic growth rate and $S_{1}, S_{2}$ are the respective food preferences of the generalist predator. Suppose that all the coefficient parameters are positive and the biological significance are given in [17]. To facilitate the calculation of reduced parameters, the following non-dimensional variables and constants were introduced:

$$
\begin{aligned}
& t=a_{0} T, \quad x=\frac{X}{K}, \quad y=\frac{a_{1}}{a_{0}} Y, \quad z=\frac{a_{2}}{a_{0}} Z, \\
& w_{1}=b_{1} K, \quad w_{2}=b_{2} K, \quad w_{3}=\frac{a_{0}}{a_{1}} b_{3}, \quad w_{4}=\frac{a_{1}}{a_{0}} c K, \quad w_{5}=\frac{r}{a_{0}}, \\
& w_{6}=\frac{a_{3}}{a_{2}}, \quad w_{7}=\frac{S_{0}}{a_{2}}, \quad w_{8}=\frac{S_{0}}{a_{2} S_{3}}, \quad w_{9}=\frac{S_{1}}{S_{3}} K, \quad w_{10}=\frac{a_{0} S_{2}}{a_{1} S_{3}} .
\end{aligned}
$$

Furthermore, system (1.1) takes the following non-dimensional form:

$$
\left\{\begin{array}{l}
\frac{d x}{d t}=x\left[1-x-\frac{y}{1+w_{1} x}-\frac{z}{1+w_{2} x+w_{3} y}\right], \\
\frac{d y}{d t}=y\left[-w_{5}+\frac{w_{4} x}{1+w_{1} x}-\frac{w_{6} z}{1+w_{2} x+w_{3} y}\right], \\
\frac{d z}{d t}=w_{7} z^{2}-\frac{w_{8} z^{2}}{1+w_{9} x+w_{10} y},
\end{array}\right.
$$

the associated initial conditions are $x(0)=x_{0} \geq 0, y(0)=y_{0} \geq 0, z(0)=z_{0} \geq 0$. When $w_{1}=1.4, w_{2}=5, w_{3}=8, w_{4}=1, w_{5}=0.16, w_{6}=0.1, w_{7}=0.1, w_{8}=0.5, w_{9}=8, w_{10}=8$, sys tem (1.2) admits a chaotic attractor (see Fig. 1). The aim of this paper is to investigate the 

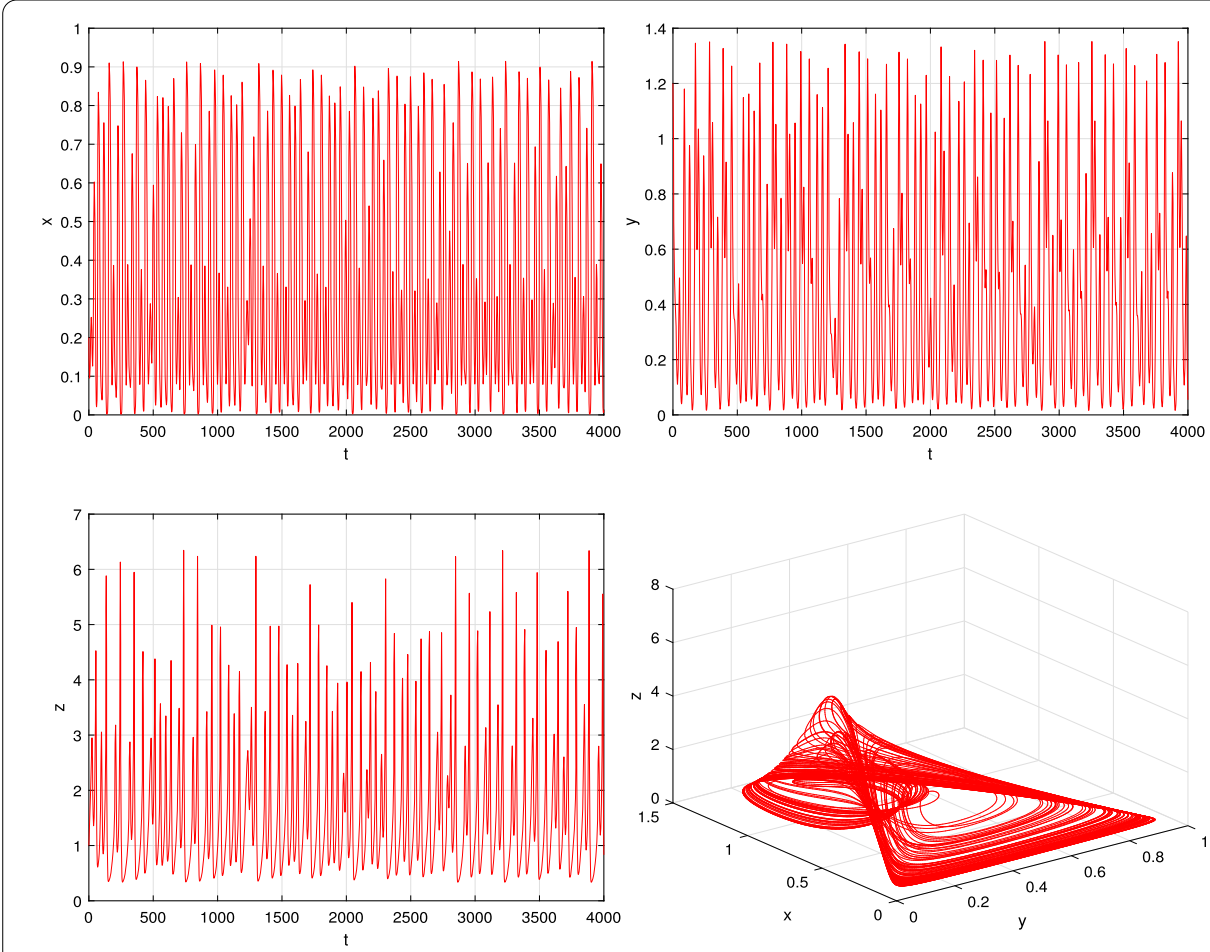

Figure 1 The trajectories and graphs of system (1.3) with $\tau=0$, where $w_{1}=1.4, w_{2}=5, w_{3}=8, w_{4}=1$, $w_{5}=0.16, w_{6}=0.1, w_{7}=0.1, w_{8}=0.5, w_{9}=8, w_{10}=8$. System (1.3) has a chaotic attractor

dynamics of model by considering the effect of delayed feedback control. Following the idea of [18], we add time-delayed force $k_{1}[x-x(t-\tau)], k_{2}[y-y(t-\tau)], k_{3}[z-z(t-\tau)]$ to system (1.2), then we have

$$
\left\{\begin{array}{l}
\frac{d x}{d t}=x\left[1-x-\frac{y}{1+w_{1} x}-\frac{z}{1+w_{2} x+w_{3} y}\right]+k_{1}[x-x(t-\tau)], \\
\frac{d y}{d t}=y\left[-w_{5}+\frac{w_{4} x}{1+w_{1} x}-\frac{w_{6} z}{1+w_{2} x+w_{3} y}\right]+k_{2}[y-y(t-\tau)], \\
\frac{d z}{d t}=w_{7} z^{2}-\frac{w_{8} z^{2}}{1+w_{9} x+w_{10} y}+k_{3}[z-z(t-\tau)] .
\end{array}\right.
$$

Regarding time delay $\tau$ as the bifurcation parameter, when $\tau$ passes through some certain critical values, the equilibrium will lose its stability and Hopf bifurcation will take place. By controlling the feedback effect strength $k_{i}(i=1,2,3)$, we can implement the control of chaos phenomena of the system. The existence of branches makes the changing laws of nature more complicated, which is also in line with objective facts. Branch theory is increasingly applied in the study of biomathematical model. It plays an important role in determining whether periodic solutions exist in biological systems [19-21].

The rest of the paper is organized as follows. In Sect. 2, the local stability and the existence of Hopf bifurcation are determined. In Sect. 3, some explicit formulas determining the direction and stability of periodic solutions bifurcating from Hopf bifurcations point are demonstrated by applying the normal form theory and the center manifold theorem. In Sect. 4, we illustrate a particular example, in which numerical simulations to verify the theorem analysis are given. Finally, there is the summary and prospect part in Sect. 5. 


\section{Bifurcation analysis of the chaotic system}

In this section, we investigate the existence of Hopf bifurcation occurring at the positive equilibrium by analyzing the characteristic equation of system (1.3). To ensure the existence of the positive of system (1.3), we assume that the coefficient of system gratifies the following conditions:

$$
\begin{aligned}
& H_{1}: w_{6}\left(w_{8}-w_{7}\right)<w_{7} w_{10}\left(w_{5}+w_{6}\right), \\
& H_{2}:\left(w_{8}-w_{7}\right)>w_{7} w_{9} x^{*}, \\
& H_{3}:\left(w_{4}-w_{1} w_{5}\right) x^{*}>w_{5} .
\end{aligned}
$$

Obviously, under hypotheses $\left(H_{1}\right),\left(H_{2}\right),\left(H_{3}\right)$, system (1.2) has a unique positive equilibrium $E_{+}\left(x^{*}, y^{*}, z^{*}\right)$,

$$
\begin{aligned}
& x^{*}=\frac{-B_{1}+\sqrt{\Delta}}{2 A_{1}}, \quad y^{*}=\frac{w_{8}-w_{7}-w_{7} w_{9} x^{*}}{w_{7} w_{10}}, \\
& z^{*}=\frac{\left(\frac{w_{4} x^{*}}{1+w_{1} x^{*}}-w_{5}\right)\left(1+w_{2} x^{*}+w_{3} y^{*}\right)}{w_{6}},
\end{aligned}
$$

where

$$
\begin{aligned}
& \Delta=B_{1}^{2}-4 A_{1} C_{1}, \quad A_{1}=w_{1} w_{6} w_{7} w_{10}, \\
& B_{1}=w_{7} w_{10}\left(w_{4}+w_{6}\right)-w_{6} w_{7} w_{9}-w_{1} w_{7} w_{10}\left(w_{5}+w_{6}\right), \\
& C_{1}=w_{6}\left(w_{8}-w_{7}\right)-w_{7} w_{10}\left(w_{5}+w_{6}\right) .
\end{aligned}
$$

When $\left(H_{1}\right),\left(H_{2}\right),\left(H_{3}\right)$ hold, the linearizing system of $(1.3)$ is

$$
\dot{u}=A u(t)+B u(t-\tau)
$$

where

$$
\begin{aligned}
& u(t)=(x, y, z)^{T}, \quad A=\left(a_{i j}\right)_{3 \times 3}, \quad B=\left(b_{i j}\right)_{3 \times 3}, \\
& a_{11}=1-2 x^{*}-\frac{y^{*}}{\left(1+w_{1} x^{*}\right)^{2}}-\frac{z^{*}\left(1+w_{3} y^{*}\right)}{\left(1+w_{2} x^{*}+w_{3} y^{*}\right)^{2}}+k_{1}, \\
& a_{12}=-\frac{x^{*}}{1+w_{1} x^{*}}+\frac{w_{3} x^{*} z^{*}}{\left(1+w_{2} x^{*}+w_{3} y^{*}\right)^{2}}, \quad a_{13}=-\frac{x^{*}}{1+w_{2} x^{*}+w_{3} y^{*}}, \\
& a_{21}=\frac{w_{4} y^{*}}{\left(1+w_{1} x^{*}\right)^{2}}+\frac{w_{2} w_{6} y^{*} z^{*}}{\left(1+w_{2} x^{*}+w_{3} y^{*}\right)^{2}}, \\
& a_{22}=-w_{5}+k_{2}+\frac{w_{4} x^{*}}{1+w_{1} x^{*}}-\frac{w_{6} z^{*}\left(1+w_{2} x^{*}\right)}{\left(1+w_{2} x^{*}+w_{3} y^{*}\right)^{2}}, \\
& a_{23}=-\frac{w_{6} y^{*}}{1+w_{2} x^{*}+w_{3} y^{*}}, \quad a_{31}=\frac{w_{8} w_{9}\left(z^{*}\right)^{2}}{\left(1+w_{9} x^{*}+w_{10} y^{*}\right)^{2}}, \\
& a_{32}=\frac{w_{8} w_{10}\left(z^{*}\right)^{2}}{\left(1+w_{9} x^{*}+w_{10} y^{*}\right)^{2}}, \quad a_{33}=2 w_{7} z^{*}-\frac{2 w_{8} z^{*}}{1+w_{9} x^{*}+w_{10} y^{*}}+k_{3},
\end{aligned}
$$




$$
b_{11}=-k_{1}, \quad b_{22}=-k_{2}, \quad b_{33}=-k_{3},
$$

all the others of $b_{i j}$ are 0 , the characteristic equation of (2.1) is

$$
\lambda^{3}+a_{2} \lambda^{2}+a_{1} \lambda+a_{0}+\left(b_{2} \lambda^{2}+b_{1} \lambda+b_{0}\right) e^{-\lambda \tau}+\left(c_{1} \lambda+c_{0}\right) e^{-2 \lambda \tau}+d_{0} e^{-3 \lambda \tau}=0,
$$

where

$$
\begin{aligned}
& a_{2}=-a_{11}-a_{22}-a_{33}, \\
& a_{1}=a_{11} a_{22}+a_{22} a_{33}+a_{11} a_{33}-a_{12} a_{21}-a_{13} a_{31}-a_{23} a_{32}, \\
& a_{0}=a_{11} a_{23} a_{32}+a_{12} a_{21} a_{33}+a_{13} a_{22} a_{31}-a_{11} a_{22} a_{33}-a_{12} a_{23} a_{31}-a_{13} a_{21} a_{32}, \\
& b_{2}=k_{1}+k_{2}+k_{3}, \quad b_{1}=-k_{1}\left(a_{22}+a_{33}\right)-k_{2}\left(a_{11}+a_{33}\right)-k_{3}\left(a_{11}+a_{22}\right), \\
& b_{0}=k_{1}\left(a_{22} a_{33}-a_{23} a_{32}\right)+k_{2}\left(a_{11} a_{33}-a_{13} a_{31}\right)+k_{3}\left(a_{11} a_{22}-a_{12} a_{21}\right), \\
& c_{1}=k_{1} k_{2}+k_{1} k_{3}+k_{2} k_{3}, \quad c_{0}=-k_{1} k_{2} a_{33}-k_{1} k_{3} a_{22}-k_{2} k_{3} a_{11}, \quad d_{0}=k_{1} k_{2} k_{3} .
\end{aligned}
$$

Multiplying $e^{\lambda \tau}$ on the both sides of Eq. (2.2), we get

$$
\left(\lambda^{3}+a_{2} \lambda^{2}+a_{1} \lambda+a_{0}\right) e^{\lambda \tau}+b_{2} \lambda^{2}+b_{1} \lambda+b_{0}+\left(c_{1} \lambda+c_{0}\right) e^{-\lambda \tau}+d_{0} e^{-2 \lambda \tau}=0
$$

For the sake of investigating the properties of the roots of the transcendental equation, we introduce the following result from [22].

\section{Lemma 1 Consider the exponential polynomial}

$$
\begin{aligned}
P(\lambda, & \left.e^{-\lambda \tau_{1}}, \ldots, e^{-\lambda \tau_{m}}\right) \\
= & \lambda^{n}+p_{1}^{(0)} \lambda^{n-1}+\cdots+p_{n-1}^{(0)} \lambda+p_{n}^{(0)} \\
& +\left[p_{1}^{(1)} \lambda^{n-1}+\cdots+p_{n-1}^{(1)} \lambda+p_{n}^{(1)}\right] e^{-\lambda \tau_{1}}+\cdots \\
& +\left[p_{1}^{(m)} \lambda^{n-1}+\cdots+p_{n-1}^{(m)} \lambda+p_{n}^{(m)}\right] e^{-\lambda \tau_{m}},
\end{aligned}
$$

where $\tau_{i} \geq 0(i=1,2, \ldots, m)$ and $p_{j}^{(i)}(i=0,1,2, \ldots, m ; j=1,2, \ldots, n)$ are constants. As $\left(\tau_{1}, \tau_{2}, \ldots, \tau_{m}\right)$ vary, the sum of the order of the zeros of $P\left(\lambda, e^{-\lambda \tau_{1}}, \ldots, e^{-\lambda \tau_{m}}\right)$ on the open right half plane can change only if a zero appears on or crosses the imaginary axis.

When $\tau=0$, then Eq. (2.3) takes the form

$$
\lambda^{3}+\left(a_{2}+b_{2}\right) \lambda^{2}+\left(a_{1}+b_{1}+c_{1}\right) \lambda+a_{0}+b_{0}+c_{0}+d_{0}=0
$$

According to the Routh-Hurwitz criterion [23], all roots of Eq. (2.4) have negative real parts provided that the following conditions are satisfied:

$$
\begin{aligned}
& \Delta_{1}=a_{2}+b_{2}>0 \\
& \Delta_{2}=\left|\begin{array}{cc}
a_{2}+b_{2} & 1 \\
a_{0}+b_{0}+c_{0}+d_{0} & a_{1}+b_{1}+c_{1}
\end{array}\right|
\end{aligned}
$$




$$
\begin{aligned}
& =\left(a_{2}+b_{2}\right)\left(a_{1}+b_{1}+c_{1}\right)-\left(a_{0}+b_{0}+c_{0}+d_{0}\right)>0 \\
\Delta_{3} & =\left|\begin{array}{ccc}
a_{2}+b_{2} & 1 & 0 \\
a_{0}+b_{0}+c_{0}+d_{0} & a_{1}+b_{1}+c_{1} & a_{2}+b_{2} \\
0 & 0 & a_{0}+b_{0}+c_{0}+d_{0}
\end{array}\right| \\
& =\left(a_{0}+b_{0}+c_{0}+d_{0}\right)\left(\left(a_{2}+b_{2}\right)\left(a_{1}+b_{1}+c_{1}\right)-\left(a_{0}+b_{0}+c_{0}+d_{0}\right)\right)>0 .
\end{aligned}
$$

That means Eq. (2.4) satisfies the following condition:

$$
\left(H_{4}\right): a_{2}+b_{2}>0, \quad\left(a_{2}+b_{2}\right)\left(a_{1}+b_{1}+c_{1}\right)>a_{0}+b_{0}+c_{0}+d_{0}, a_{0}+b_{0}+c_{0}+d_{0}>0
$$

Then the positive equilibrium is locally asymptotically stable when $\tau=0$.

Obviously, Eq. (2.3) has a pair of purely imaginary roots $\pm i \omega_{0}\left(\omega_{0}>0\right)$ if and only if $\omega_{0}$ satisfies

$$
\begin{gathered}
\left(-i \omega_{0}^{3}-a_{2} \omega_{0}^{2}+a_{1} i \omega_{0}+a_{0}\right) e^{i \omega_{0} \tau}-b_{2} \omega_{0}^{2}+b_{1} i \omega_{0}+b_{0} \\
+\left(c_{1} i \omega_{0}+c_{0}\right) e^{-i \omega_{0} \tau}+d_{0} e^{-2 i \omega_{0} \tau}=0 .
\end{gathered}
$$

Separating the real and imaginary parts, we have

$$
\begin{aligned}
& m_{1} \cos \omega_{0} \tau+m_{2} \sin \omega_{0} \tau+m_{3}=-d_{0} \cos 2 \omega_{0} \tau, \\
& n_{1} \cos \omega_{0} \tau+n_{2} \sin \omega_{0} \tau+n_{3}=d_{0} \sin 2 \omega_{0} \tau,
\end{aligned}
$$

where

$$
\begin{aligned}
& m_{1}=a_{0}-a_{2} \omega_{0}^{2}+c_{0}, \quad m_{2}=c_{1} \omega_{0}-a_{1} \omega_{0}+\omega_{0}^{3}, \quad m_{3}=b_{0}-b_{2} \omega_{0}^{2} \text {, } \\
& n_{1}=a_{1} \omega_{0}-\omega_{0}^{3}+c_{1} \omega_{0}, \quad n_{2}=a_{0}-a_{2} \omega_{0}^{2}-c_{0}, \quad n_{3}=b_{1} \omega_{0} \text {. }
\end{aligned}
$$

It follows from (2.6) that

$$
\begin{aligned}
& \left(m_{1}^{2}+n_{1}^{2}\right) \cos ^{2} \omega_{0} \tau+\left(m_{2}^{2}+n_{2}^{2}\right) \sin ^{2} \omega_{0} \tau+2\left(m_{1} m_{2}+n_{1} n_{2}\right) \sin \omega_{0} \tau \cos \omega_{0} \tau \\
& \quad+2\left(m_{1} m_{3}+n_{1} n_{3}\right) \cos \omega_{0} \tau+2\left(m_{2} m_{3}+n_{2} n_{3}\right) \sin \omega_{0} \tau+m_{3}^{2}+n_{3}^{2}-d_{0}^{2}=0 .
\end{aligned}
$$

In view of $\sin \omega_{0} \tau= \pm \sqrt{1-\cos ^{2} \omega_{0} \tau}$ and (2.7), we get

$$
q_{1} \cos ^{4} \omega_{0} \tau+q_{2} \cos ^{3} \omega_{0} \tau+q_{3} \cos ^{2} \omega_{0} \tau+q_{4} \cos \omega_{0} \tau+q_{5}=0
$$

where

$$
\begin{aligned}
q_{1}= & \left(m_{1}^{2}+n_{1}^{2}-m_{2}^{2}-n_{2}^{2}\right)^{2}+4\left(m_{1} m_{2}+n_{1} n_{2}\right)^{2}, \\
q_{2}= & 4\left(m_{1}^{2}+n_{1}^{2}-m_{2}^{2}-n_{2}^{2}\right)\left(m_{1} m_{3}+n_{1} n_{3}\right)+8\left(m_{1} m_{2}+n_{1} n_{2}\right)\left(m_{2} m_{3}+n_{2} n_{3}\right), \\
q_{3}= & 2\left(m_{1}^{2}+n_{1}^{2}-m_{2}^{2}-n_{2}^{2}\right)\left(m_{2}^{2}+n_{2}^{2}+m_{3}^{2}+n_{3}^{2}-d_{0}^{2}\right)+4\left(m_{1} m_{3}+n_{1} n_{3}\right)^{2} \\
& +4\left(m_{2} m_{3}+n_{2} n_{3}\right)^{2}-4\left(m_{1} m_{2}+n_{1} n_{2}\right)^{2},
\end{aligned}
$$




$$
\begin{aligned}
q_{4}= & 4\left(m_{1} m_{3}+n_{1} n_{3}\right)\left(m_{2}^{2}+n_{2}^{2}+m_{3}^{2}+n_{3}^{2}-d_{0}^{2}\right) \\
& -8\left(m_{1} m_{2}+n_{1} n_{2}\right)\left(m_{2} m_{3}+n_{2} n_{3}\right), \\
q_{5}= & \left(m_{2}^{2}+n_{2}^{2}+m_{3}^{2}+n_{3}^{2}-d_{0}^{2}\right)-4\left(m_{2} m_{3}+n_{2} n_{3}\right)^{2} .
\end{aligned}
$$

Let $\cos \omega_{0} \tau=r$ and denote

$$
h(r)=r^{4}+\frac{q_{2}}{q_{1}} r^{3}+\frac{q_{3}}{q_{1}} r^{2}+\frac{q_{4}}{q_{1}} r+\frac{q_{5}}{q_{1}}
$$

then

$$
h^{\prime}(r)=4 r^{3}+3 \frac{q_{2}}{q_{1}} r^{2}+2 \frac{q_{3}}{q_{1}} r+\frac{q_{4}}{q_{1}} .
$$

Set

$$
4 r^{3}+3 \frac{q_{2}}{q_{1}} r^{2}+2 \frac{q_{3}}{q_{1}} r+\frac{q_{4}}{q_{1}}=0
$$

Let $r=z-\frac{q_{2}}{4 q_{1}}$. Then Eq. (2.8) takes the form

$$
z^{3}+\rho_{1} z+\rho_{2}=0
$$

where $\rho_{1}=\frac{q_{3}}{2 q_{2}}-\frac{3 q_{2}^{2}}{16 q_{1}^{2}}, \rho_{2}=\frac{q_{2}^{3}}{32 q_{1}^{3}}-\frac{q_{2} q_{3}}{8 q_{1}^{2}}+\frac{q_{4}}{4 q_{1}}$.

Define $\sigma_{1}=\left(\frac{\rho_{2}}{2}\right)^{2}+\left(\frac{\rho_{1}}{3}\right)^{3}, \sigma_{2}=\frac{-1+\sqrt{3} i}{2}, \sigma_{3}=\frac{-1-\sqrt{3} i}{2}$. By Eq. (2.9), we obtain

$$
\begin{aligned}
& z_{1}=\sqrt[3]{-\frac{\rho_{2}}{2}+\sqrt{\sigma_{1}}}+\sqrt[3]{-\frac{\rho_{2}}{2}-\sqrt{\sigma_{1}}}, \\
& z_{2}=\sigma_{2} \sqrt[3]{-\frac{\rho_{2}}{2}+\sqrt{\sigma_{1}}}+\sigma_{3} \sqrt[3]{-\frac{\rho_{2}}{2}-\sqrt{\sigma_{1}}}, \\
& z_{3}=\sigma_{3} \sqrt[3]{-\frac{\rho_{2}}{2}+\sqrt{\sigma_{1}}}+\sigma_{2} \sqrt[3]{-\frac{\rho_{2}}{2}-\sqrt{\sigma_{1}}} .
\end{aligned}
$$

Based on the analysis and calculation above, we can deduce the expression of $\cos \omega_{0} \tau$, i.e.,

$$
\cos \omega_{0} \tau=f_{1}\left(\omega_{0}\right)
$$

where $f_{1}\left(\omega_{0}\right)$ is a function with respect to $\omega_{0}$. Substituting (2.10) into (2.7), we can easily get the expression of $\sin \omega_{0} \tau$, i.e.,

$$
\sin \omega_{0} \tau=f_{2}\left(\omega_{0}\right)
$$

where $f_{2}\left(\omega_{0}\right)$ is a function with respect to $\omega_{0}$. Thus we get

$$
f_{1}\left(\omega_{0}\right)^{2}+f_{2}\left(\omega_{0}\right)^{2}=1
$$


Through a series of calculations, we can easily calculate Eq. (2.12). Then it follows from (2.10) that

$$
\tau_{k}=\frac{1}{\omega_{0}}\left[\arccos f_{1}\left(\omega_{0}\right)+2 k \pi\right] \quad(k=0,1,2, \ldots) .
$$

Clearly, when $\tau=\tau_{k}(k=0,1,2, \ldots)$ Eq. (2.3) has a simple pair of imaginary roots $\pm i \omega_{0}$. Concluding the above analysis, and according to Lemma 1 and [24], we can obtain the following Lemma 2.

Lemma 2 Let $\tau_{k}$ be defined by (2.13). If $\left(H_{1}\right)-\left(H_{4}\right)$ hold, then we have the following:

(i) When $\tau=\tau_{k}$,Eq. (2.3) has a simple pair of imaginary roots $\pm i \omega_{0}$, where $\omega_{0}$ is the positive root of Eq. (2.12).

(ii) When $\tau \in\left[0, \tau_{0}\right)$, all the roots of $E q$. (2.14) have strictly negative real parts.

(iii) When $\tau=\tau_{k}$, Eq. (2.3) has a pair of imaginary roots $\pm i \omega$ and all other roots have strictly negative real parts.

Let $\lambda(\tau)=\alpha(\tau)+i \omega(\tau)$ be the root of the characteristic Eq. (2.2) near $\tau=\tau_{k}$, satisfying $\alpha\left(\tau_{k}\right)=0, \omega\left(\tau_{k}\right)=\omega_{0}(k=0,1,2, \ldots)$. Substituting $\lambda(\tau)$ into Eq. (2.2) and taking the derivative with respect to $\tau$, we obtain

$$
\begin{aligned}
& {\left[\left(3 \lambda^{2}+2 a_{2} \lambda+a_{1}\right) e^{\lambda \tau}+\tau\left(\lambda^{3}+a_{2} \lambda^{2}+a_{1} \lambda+a_{0}\right) e^{\lambda \tau}+2 b_{2} \lambda+b_{1}+c_{1} e^{-\lambda \tau}\right.} \\
& \left.\quad-\tau\left(c_{1} \lambda+c_{0}\right) e^{-\lambda \tau}-2 \tau d_{0} e^{-2 \lambda \tau}\right] \frac{d \lambda}{d \tau}+\lambda\left(\lambda^{3}+a_{2} \lambda^{2}+a_{1} \lambda+a_{0}\right) e^{\lambda \tau} \\
& \quad-\lambda\left(c_{1} \lambda+c_{0}\right) e^{-\lambda \tau}-2 \lambda d_{0} e^{-2 \lambda \tau}=0
\end{aligned}
$$

hence

$$
\left[\frac{d \lambda}{d \tau}\right]^{-1}=\frac{\left(3 \lambda^{2}+2 a_{2} \lambda+a_{1}\right) e^{\lambda \tau}+2 b_{2} \lambda+b_{1}+c_{1} e^{-\lambda \tau}}{-\lambda\left(\lambda^{3}+a_{2} \lambda^{2}+a_{1} \lambda+a_{0}\right) e^{\lambda \tau}+\left(c_{1} \lambda+c_{0}\right) \lambda e^{-\lambda \tau}+2 d_{0} \lambda e^{-2 \lambda \tau}}-\frac{\tau}{\lambda} .
$$

Then

$$
\operatorname{Re}\left[\frac{d \lambda}{d \tau}\right]_{\tau=\tau_{k}}^{-1}=\operatorname{Re}\left[\frac{N_{1}+i N_{2}}{M_{1}+i M_{2}}\right]=\frac{M_{1} N_{1}+M_{2} N_{2}}{M_{1}^{2}+M_{2}^{2}}
$$

where

$$
\begin{aligned}
M_{1}= & \omega_{0}^{2}\left(-\omega_{0}^{2}+a_{1}-c_{1}\right) \cos \omega_{0} \tau_{k}+\omega_{0}\left(c_{0}+a_{0}-a_{2} \omega_{0}^{2}\right) \sin \omega_{0} \tau_{k} \\
& +2 d_{0} \omega_{0} \sin 2 \omega_{0} \tau_{k}, \\
M_{2}= & \omega_{0}^{2}\left(-\omega_{0}^{2}+a_{1}+c_{1}\right) \sin \omega_{0} \tau_{k}+\omega_{0}\left(c_{0}-a_{0}+a_{2} \omega_{0}^{2}\right) \cos \omega_{0} \tau_{k} \\
& +2 d_{0} \omega_{0} \cos 2 \omega_{0} \tau_{k}, \\
N_{1}= & \left(a_{1}+c_{1}-3 \omega_{0}^{2}\right) \cos \omega_{0} \tau_{k}-2 a_{2} \omega_{0} \sin \omega_{0} \tau_{k}+b_{1}, \\
N_{2}= & \left(a_{1}-c_{1}-3 \omega_{0}^{2}\right) \sin \omega_{0} \tau_{k}+2 a_{2} \omega_{0} \cos \omega_{0} \tau_{k}+2 b_{2} \omega_{0} .
\end{aligned}
$$


Lemma 3 Let $\tau=\tau_{k}$, and suppose condition $\left(H_{5}\right): M_{1} N_{1}+M_{2} N_{2} \neq 0$ holds, then the following transversality condition

$$
\left.\frac{d \operatorname{Re}[\lambda(\tau)]}{d \tau}\right|_{\tau=\tau_{k}} \neq 0
$$

is satisfied.

On the basis of previous analysis and the results of [25] and [26], we can easily obtain the following theorem.

Theorem 1 If conditions $\left(H_{1}\right)-\left(H_{5}\right)$ hold, $\tau_{k}$ is defined by (2.13). Then the positive equilibrium of system (1.3) is asymptotically stable for $\tau \in\left[0, \tau_{0}\right)$ and system (1.3) undergoes a Hopf bifurcation at the positive equilibrium when $\tau=\tau_{k}(k=0,1,2, \ldots)$.

\section{Direction and stability of Hopf bifurcation period solution}

In this section, we investigate the direction of Hopf bifurcation and the stability of the bifurcating periodic solutions based on the normal form theory and center manifold theorem introduced by [27]. We assume that system (1.2) always undergoes Hopf bifurcation at the positive equilibrium $E_{+}$for $\tau=\tau_{k}^{j}(k=1,2$ and $j=0,1,2, \ldots)$ and the characteristic Eq. (2.3) has a pair of purely imaginary roots at the positive equilibrium $E_{+}$.

Let $x_{1}=x-x_{0}, x_{2}=y-y_{0}, x_{3}=z-z_{0}, \bar{x}_{i}=x_{i}(t \tau), \tau=\tau_{k}+\mu$, dropping the bars for simplification of notations, and let $p(x)=\frac{x}{1+w_{1} x}, q_{1}(x, y)=\frac{x}{1+w_{2} x+w_{3} y}, q_{2}(x, y)=\frac{y}{1+w_{2} x+w_{3} y}$, $R(x, y)=\frac{1}{1+w_{9} x+w_{10} y}$. Then the nonlinear system (1.3) can be transformed into a functional differential equation (FDE) system in $C \in C\left([-1,0], R^{3}\right)$ as

$$
\dot{x}(t)=L_{\mu}\left(x_{t}\right)+f\left(\mu, x_{t}\right)
$$

where $x(t)=\left(x_{1}(t), x_{2}(t), x_{3}(t)\right)^{T} \in R^{3}$ and $L_{\mu}: C \rightarrow R^{3}, f: R \times C \rightarrow R^{3}$ are given respectively by

$$
\begin{aligned}
L_{\mu}(\varphi)= & \left(\mu+\tau^{j}\right)\left(\begin{array}{lll}
a_{11} & a_{12} & a_{13} \\
a_{21} & a_{22} & a_{23} \\
a_{31} & a_{32} & a_{33}
\end{array}\right)\left(\begin{array}{l}
\varphi_{1}(0) \\
\varphi_{2}(0) \\
\varphi_{3}(0)
\end{array}\right) \\
& +\left(\mu+\tau_{k}\right)\left(\begin{array}{ccc}
b_{11} & 0 & 0 \\
0 & b_{22} & 0 \\
0 & 0 & b_{33}
\end{array}\right)\left(\begin{array}{l}
\varphi_{1}(-1) \\
\varphi_{2}(-1) \\
\varphi_{3}(-1)
\end{array}\right)
\end{aligned}
$$

and

$$
f(\mu, \varphi)=\left(\mu+\tau_{k}\right)\left(\begin{array}{l}
f_{1} \\
f_{2} \\
f_{3}
\end{array}\right),
$$

where

$$
f_{1}=-l_{11} \varphi_{1}^{2}(0)-l_{12} \varphi_{1}(0) \varphi_{2}(0)-l_{13} \varphi_{1}(0) \varphi_{3}(0)-l_{14} \varphi_{2}(0) \varphi_{3}(0)
$$




$$
\begin{aligned}
& -l_{15} \varphi_{1}^{2}(0) \varphi_{2}(0)-l_{16} \varphi_{1}^{3}(0)-l_{17} \varphi_{1}^{3}(0) \varphi_{2}(0)-l_{18} \varphi_{2}^{2}(0) \\
& -l_{19} \varphi_{2}^{3}(0)-l_{110} \varphi_{2}^{2}(0) \varphi_{3}(0)+\cdots, \\
f_{2}= & l_{21} \varphi_{1}^{2}(0)+l_{22} \varphi_{1}(0) \varphi_{2}(0)+l_{23} \varphi_{1}^{3}(0)+l_{24} \varphi_{1}^{3}(0) \varphi_{2}(0) \\
& +l_{25} \varphi_{1}^{2}(0) \varphi_{2}(0)-l_{26} \varphi_{1}(0) \varphi_{3}(0)-l_{27} \varphi_{2}(0) \varphi_{3}(0)-l_{28} \varphi_{1}^{2}(0) \varphi_{3}(0) \\
& -l_{29} \varphi_{2}^{2}(0) \varphi_{3}(0)-l_{210} \varphi_{2}^{3}(0)-l_{211} \varphi_{2}^{3}(0) \varphi_{3}(0)-l_{212} \varphi_{1}^{2}(0) \varphi_{2}^{2}(0) \\
& -l_{213} \varphi_{1}(0) \varphi_{2}^{3}(0)+\cdots, \\
f_{3}= & l_{31} \varphi_{3}^{2}(0)-l_{32} \varphi_{1}^{2}(0)-l_{33} \varphi_{2}^{2}(0)-l_{34} \varphi_{1}(0) \varphi_{2}(0)-l_{35} \varphi_{1}(0) \varphi_{3}(0) \\
& -l_{36} \varphi_{2}(0) \varphi_{3}(0)-l_{37} \varphi_{1}^{3}(0)-l_{38} \varphi_{2}^{3}(0)-l_{39} \varphi_{1}(0) \varphi_{2}(0) \varphi_{3}(0) \\
& -l_{310} \varphi_{1}^{2}(0) \varphi_{2}(0)-l_{311} \varphi_{2}^{2}(0) \varphi_{1}(0)-l_{312} \varphi_{1}(0) \varphi_{3}^{2}(0) \\
& -l_{313} \varphi_{2}(0) \varphi_{3}^{2}(0)-l_{314} \varphi_{1}^{2}(0) \varphi_{3}(0)-l_{315} \varphi^{2}(0) \varphi_{3}(0)+\cdots
\end{aligned}
$$

and

$$
\begin{aligned}
& \varphi(\theta)=\left(\varphi_{1}(\theta), \varphi_{2}(\theta), \varphi_{3}(\theta)\right)^{T} \in R^{3}, \\
& l_{11}=1+\frac{1}{2} p^{\prime \prime}\left(x^{*}\right) y^{*}+\frac{1}{2} q_{1}^{\prime \prime}\left(x^{*}\right) z^{*}, \quad l_{12}=p^{\prime}\left(x^{*}\right)+q_{1}^{\prime}\left(x^{*}\right) q_{1}^{\prime}\left(y^{*}\right) z^{*}, \\
& l_{13}=q_{1}^{\prime}\left(x^{*}\right), \quad l_{14}=q_{1}^{\prime}\left(y^{*}\right), \quad l_{15}=\frac{1}{2} p^{\prime \prime}\left(x^{*}\right)+\frac{1}{2} q_{1}^{\prime \prime}\left(x^{*}\right) q_{1}^{\prime}\left(y^{*}\right) z^{*}, \\
& l_{16}=\frac{1}{6} p^{\prime \prime \prime}\left(x^{*}\right) y^{*}+\frac{1}{6} q_{1}^{\prime \prime \prime}\left(x^{*}\right) z^{*}, \quad l_{17}=\frac{1}{6} p^{\prime \prime \prime}\left(x^{*}\right)+\frac{1}{6} q_{1}^{\prime \prime \prime}\left(x^{*}\right) q_{1}^{\prime}\left(y^{*}\right) z^{*}, \\
& l_{18}=\frac{1}{2} q_{1}^{\prime \prime}\left(y^{*}\right) z^{*}, \quad l_{19}=\frac{1}{6} q_{1}^{\prime \prime \prime}\left(y^{*}\right) z^{*}, \quad l_{110}=\frac{1}{2} q_{1}^{\prime \prime}\left(y^{*}\right), \\
& l_{21}=\frac{w_{4}}{2} p^{\prime \prime}\left(x^{*}\right) y^{*}-\frac{w_{6}}{2} q_{2}^{\prime \prime}\left(x^{*}\right) z^{*}, \\
& l_{22}=w_{4} p^{\prime}\left(x^{*}\right)-w_{6} q_{2}^{\prime}\left(x^{*}\right) q_{2}^{\prime}\left(y^{*}\right) z^{*}, \\
& l_{23}=\frac{w_{4}}{6} p^{\prime \prime \prime}\left(x^{*}\right) y^{*}-\frac{w_{6}}{6} q_{2}^{\prime \prime \prime}\left(x^{*}\right) z^{*}, \\
& l_{24}=\frac{w_{4}}{6} p^{\prime \prime \prime}\left(x^{*}\right)-\frac{w_{6}}{6} q_{2}^{\prime \prime \prime}\left(x^{*}\right) q_{2}^{\prime}\left(y^{*}\right) z^{*}, \\
& l_{25}=\frac{w_{4}}{2} p^{\prime \prime}\left(x^{*}\right)-\frac{w_{6}}{2} q_{2}^{\prime \prime}\left(x^{*}\right) q_{2}^{\prime}\left(y^{*}\right) z^{*}, \\
& l_{26}=w_{6} q_{2}^{\prime}\left(x^{*}\right), \quad l_{27}=w_{6} q_{2}^{\prime}\left(y^{*}\right), \quad l_{28}=\frac{w_{6}}{2} q_{2}^{\prime \prime}\left(x^{*}\right), \quad l_{29}=\frac{w_{6}}{2} q_{2}^{\prime \prime}\left(y^{*}\right), \\
& l_{210}=\frac{w_{6}}{6} q_{2}^{\prime \prime \prime}\left(y^{*}\right) z^{*}, \quad l_{211}=\frac{w_{6}}{6} q_{2}^{\prime \prime \prime}\left(y^{*}\right), \quad l_{212}=\frac{w_{6}}{4} q_{2}^{\prime \prime}\left(x^{*}\right) q_{2}^{\prime \prime}\left(y^{*}\right) z^{*}, \\
& l_{213}=\frac{w_{6}}{6} q_{2}^{\prime}\left(x^{*}\right) q_{2}^{\prime \prime \prime}\left(y^{*}\right) z^{*}, \quad l_{31}=w_{7}-w_{8} R\left(x^{*}, y^{*}\right), \\
& l_{32}=\frac{w_{8}}{2} R_{x}^{\prime \prime}\left(x^{*}, y^{*}\right)\left(z^{*}\right)^{2}, \quad l_{33}=\frac{w_{8}}{2} R_{y}^{\prime \prime}\left(x^{*}, y^{*}\right)\left(z^{*}\right)^{2}, \\
& l_{34}=w_{8}\left(R^{\prime}\left(x^{*}, y^{*}\right)\right)^{2}\left(z^{*}\right)^{2}, \quad l_{35}=2 w_{8} R_{x}^{\prime}\left(x^{*}, y^{*}\right) z^{*}, \\
& l_{36}=2 w_{8} R_{y}^{\prime}\left(x^{*}, y^{*}\right) z^{*}, \quad l_{37}=\frac{w_{8}}{6} R_{x}^{\prime \prime \prime}\left(x^{*}, y^{*}\right)\left(z^{*}\right)^{2}, \\
& l_{38}=\frac{w_{8}}{6} R_{y}^{\prime \prime \prime}\left(x^{*}, y^{*}\right)\left(z^{*}\right)^{2}, \quad l_{39}=2 w_{8} R_{x}^{\prime}\left(x^{*}, y^{*}\right) R_{y}^{\prime}\left(x^{*}, y^{*}\right) z^{*},
\end{aligned}
$$




$$
\begin{aligned}
& l_{310}=\frac{w_{8}}{2} R_{x}^{\prime \prime}\left(x^{*}, y^{*}\right) R_{y}^{\prime}\left(x^{*}, y^{*}\right)\left(z^{*}\right)^{2}, \\
& l_{311}=\frac{w_{8}}{2} R_{x}^{\prime}\left(x^{*}, y^{*}\right) R_{y}^{\prime \prime}\left(x^{*}, y^{*}\right)\left(z^{*}\right)^{2}, \\
& l_{312}=w_{8} R_{x}^{\prime}\left(x^{*}, y^{*}\right), \quad l_{313}=w_{8} R_{y}^{\prime}\left(x^{*}, y^{*}\right), \quad l_{314}=w_{8} R_{x}^{\prime \prime}\left(x^{*}, y^{*}\right) z^{*}, \\
& l_{315}=w_{8} R_{y}^{\prime \prime}\left(x^{*}, y^{*}\right) z^{*} .
\end{aligned}
$$

By the Riesz representation theorem, there exists a function $\eta(\theta, \mu)$ of bounded variation for $\theta \in[-1,0]$. If we choose

$$
\begin{aligned}
\eta(\theta, \mu)= & \left(\mu+\tau_{k}\right)\left(\begin{array}{lll}
a_{11} & a_{12} & a_{13} \\
a_{21} & a_{22} & a_{23} \\
a_{31} & a_{32} & a_{33}
\end{array}\right) \delta(\theta) \\
& -\left(\mu+\tau_{k}\right)\left(\begin{array}{ccc}
b_{11} & 0 & 0 \\
0 & b_{22} & 0 \\
0 & 0 & b_{33}
\end{array}\right) \delta(\theta+1),
\end{aligned}
$$

where $\delta(\cdot)$ is a Dirac delta function and $\theta \in[-1,0]$, then

$$
L_{\mu}(\varphi)=\int_{-1}^{0} d \eta(\theta, \mu) \varphi(\theta) \quad \text { for } \varphi \in C
$$

For $\varphi \in C\left([-1,0], R^{3}\right)$, define

$$
A(\mu) \varphi= \begin{cases}\frac{d \varphi(\theta)}{d \theta}, & \theta \in[-1,0) \\ \int_{-1}^{0} d \eta(\mu, s) \varphi(s), & \theta=0\end{cases}
$$

and

$$
R(\mu) \varphi= \begin{cases}0, & \theta \in[-1,0) \\ f(\mu, \varphi), & \theta=0\end{cases}
$$

For convenience, we can write system (3.1) into an operate

$$
\dot{x}(t)=A(\mu) x_{t}+R(\mu) x_{t},
$$

where $x_{t}(\theta)=x(t+\theta), \theta \in[-1,0]$. For $\psi \in C^{1}\left([0,1],\left(R^{3}\right)^{*}\right)$, define

$$
A^{*} \psi(s)= \begin{cases}-\frac{d \psi(s)}{d s}, & s \in(0,1] \\ \int_{-1}^{0} d \eta^{T}(t, 0) \psi(-t), & s=0,\end{cases}
$$

and a bilinear inner product

$$
\langle\psi(s), \varphi(\theta)\rangle=\bar{\psi}(0) \varphi(0)-\int_{-1}^{0} \int_{\xi=0}^{\theta} \bar{\psi}(\xi-\theta) d \eta(\theta) \varphi(\xi) d \xi,
$$


where $\eta(\theta)=\eta(\theta, 0)$. Obviously $A^{*}$ and $A(0)$ are adjoint operators. By the discussion in Sect. 2, we know that $\pm i \omega_{0} \tau_{k}$ are eigenvalues of $A(0)$. Thus they are also eigenvalues of $A^{*}$. We need to calculate the eigenvectors of $A^{*}$ and $A(0)$ corresponding to $-i \omega_{0} \tau_{k}$ and $i \omega_{0} \tau_{k}$, respectively. Let $q(\theta)=(1, \alpha, \beta)^{T} e^{i \theta \omega_{0} \tau_{k}}$ be the eigenvector of $A(0)$, in other words, $A(0) q(\theta)=i \omega_{0} \tau_{k} q(\theta)$. Then we have

$$
\begin{aligned}
& \tau_{k}\left(\begin{array}{ccc}
i \omega_{0}-a_{11}+k_{1} e^{-i \omega_{0} \tau_{k}} & -a_{12} & -a_{13} \\
-a_{21} & i \omega_{0}-a_{22}+k_{2} e^{-i \omega_{0} \tau_{k}} & -a_{23} \\
-a_{31} & -a_{32} & i \omega_{0}-a_{33}+k_{3} e^{-i \omega_{0} \tau_{k}}
\end{array}\right), \\
& q(0)=\left(\begin{array}{l}
0 \\
0 \\
0
\end{array}\right) .
\end{aligned}
$$

Then it is easy to obtain

$$
\begin{aligned}
q(0)=(1, \alpha, \beta)^{T}= & \left(1, \frac{a_{13} a_{21}-a_{11} a_{23}+i a_{23} \omega_{0}+a_{23} k_{1} e^{-i \omega_{0} \tau_{k}}}{a_{12} a_{23}-a_{13} a_{22}+i a_{13} \omega_{0}+a_{13} k_{2} e^{-i \omega_{0} \tau_{k}}},\right. \\
& \left.\frac{a_{12} a_{31}-a_{11} a_{32}+i a_{32} \omega_{0}+a_{32} k_{1} e^{-i \omega_{0} \tau_{k}}}{a_{13} a_{32}-a_{12} a_{33}+i a_{12} \omega_{0}+a_{12} k_{3} e^{-i \omega_{0} \tau_{k}}}\right)^{T} .
\end{aligned}
$$

Similarly, we suppose that $q^{*}(s)=D\left(1, \alpha^{*}, \beta^{*}\right) e^{i s \omega_{k} \tau_{k}}$. From the definition of $A^{*}$, we have

$$
\begin{aligned}
q^{*}(s)= & D\left(1, \frac{a_{12} a_{31}-a_{11} a_{32}+i a_{32} \omega_{0}+a_{32} k_{1} e^{-i \omega_{0} \tau_{k}}}{a_{21} a_{32}-a_{22} a_{31}-i a_{31} \omega_{0}+a_{31} k_{2} e^{-i \omega_{0} \tau_{k}}},\right. \\
& \left.\frac{a_{13} a_{21}-a_{11} a_{23}+i a_{23} \omega_{0}+a_{23} k_{1} e^{-i \omega_{0} \tau_{k}}}{a_{23} a_{31}-a_{21} a_{33}-i a_{21} \omega_{0}+a_{21} k_{3} e^{-i \omega_{0} \tau_{k}}}\right) e^{i s \omega_{k} \tau_{k}},
\end{aligned}
$$

where $D$ is a constant such that $\left\langle q^{*}(s), q(\theta)\right\rangle=1$, and according to (3.7), we get

$$
\begin{aligned}
\left\langle q^{*}(s), q(\theta)\right\rangle= & \bar{D}\left(1, \bar{\alpha}^{*}, \bar{\beta}^{*}\right)(1, \alpha, \beta)^{T} \\
& -\int_{-1}^{0} \int_{\xi=0}^{\theta} \bar{D}\left(1, \overline{\alpha^{*}}, \overline{\beta^{*}}\right) e^{-i(\xi-\theta) \omega_{0} \tau_{k}} d \eta(\theta)(1, \alpha, \beta)^{T} e^{i \xi \omega_{0} \tau_{k}} d \xi \\
= & \bar{D}\left[1+\bar{\alpha}^{*} \alpha+\bar{\beta}^{*} \beta-\int_{-1}^{0}\left(1, \bar{\alpha}^{*}, \bar{\beta}^{*}\right) \theta e^{i \theta \omega_{0} \tau_{k}} d \eta(\theta)(1, \alpha, \beta)^{T}\right] \\
= & \bar{D}\left[1+\bar{\alpha}^{*} \alpha+\bar{\beta}^{*} \beta-\left(k_{1}+k_{2} \bar{\alpha}^{*} \alpha+k_{3} \bar{\beta}^{*} \beta\right) \tau_{0} e^{-i \omega_{0} \tau_{k}}\right] .
\end{aligned}
$$

Therefore, we can choose $\bar{D}$ as

$$
\bar{D}=\frac{1}{1+\bar{\alpha}^{*} \alpha+\bar{\beta}^{*} \beta-\left(k_{1}+k_{2} \bar{\alpha}^{*} \alpha+k_{3} \bar{\beta}^{*} \beta\right) \tau_{0} e^{-i \omega_{0} \tau_{k}}} .
$$

Following the ideas in [27] and using the same notation as in [28] to compute the coordinates describing the center manifold $C_{0}$ at $\mu=0$, let $x_{t}$ be the solution of Eq. (3.1) when $\mu=0$.

Define

$$
z(t)=\left\langle q^{*}, x_{t}\right\rangle, \quad W(t, \theta)=x_{t}(\theta)-2 \operatorname{Re}\{z(t) q(\theta)\} .
$$


On the center manifold $C_{0}$, we get

$$
W(t, \theta)=W(z(t), \bar{z}(t), \theta)
$$

where

$$
W(z(t), \bar{z}(t), \theta)=W_{20}(\theta) \frac{z^{2}}{2}+W_{11}(\theta) z \bar{z}+W_{02}(\theta) \frac{\bar{z}^{2}}{2}+W_{30}(\theta) \frac{z^{3}}{6}+\cdots
$$

and $z$ and $\bar{z}$ are local coordinates for the center manifold $C_{0}$ in the direction of $q^{*}$ and $\bar{q}^{*}$. Noting that $\mathrm{W}$ is also real if $x_{t} \in C_{0}$, since $\mu=0$, we have

$$
\begin{aligned}
\dot{z} & =\left\langle q^{*}(s), \dot{x}_{t}\right\rangle \\
& =\left\langle q^{*}(s), A(0) x_{t}+R(0) x_{t}\right\rangle=\left\langle q^{*}(s), A(0) x_{t}\right\rangle+\left\langle q^{*}(s), R(0) x_{t}\right\rangle \\
& =\left\langle A^{*} q^{*}(s), x_{t}\right\rangle+\bar{q}^{*}(0) R(0) x_{t}-\int_{-1}^{0} \int_{\xi=0}^{\theta} \overline{q^{*}}(\xi-\theta) d \eta(\theta) A(0) R(0) x_{t}(\xi) d \xi \\
& =\left\langle i \omega_{0} \tau_{k} q^{*}(s), x_{t}\right\rangle+\bar{q}^{*}(0) f\left(0, x_{t}(\theta)\right) \\
& =i \omega_{0} \tau_{k} z(t)+\bar{q}^{*}(0) f_{0}(z(t), \bar{z}(t)) .
\end{aligned}
$$

That is,

$$
\dot{z}(t)=i \omega_{0} \tau_{k} z+g(z, \bar{z})
$$

where

$$
g(z, \bar{z})=\bar{q}^{*}(0) f_{0}(z, \bar{z})=g_{20} \frac{z^{2}}{2}+g_{11} z \bar{z}+g_{02} \frac{\bar{z}^{2}}{2}+g_{21} \frac{z^{2} \bar{z}}{2}+\cdots
$$

Noticing

$$
\begin{aligned}
& x_{t}(\theta)=\left(x_{1 t}(\theta), x_{2 t}(\theta), x_{3 t}(\theta)\right)^{T}=W(t, \theta)+z q(\theta)+\bar{z} \bar{q}(\theta), \\
& q(\theta)=(1, \alpha, \beta)^{T} e^{i \omega_{0} \theta}
\end{aligned}
$$

we have

$$
\begin{aligned}
& x_{1 t}(0)=z+\bar{z}+W_{20}^{(1)}(0) \frac{z^{2}}{2}+W_{11}^{(1)}(0) z \bar{z}+W_{02}^{(1)}(0) \frac{\bar{z}^{2}}{2}+\cdots \\
& x_{2 t}(0)=\alpha z+\bar{\alpha} \bar{z}+W_{20}^{(2)}(0) \frac{z^{2}}{2}+W_{11}^{(2)}(0) z \bar{z}+W_{02}^{(2)}(0) \frac{\bar{z}^{2}}{2}+\cdots \\
& x_{3 t}(0)=\beta z+\bar{\beta} \bar{z}+W_{20}^{(3)}(0) \frac{z^{2}}{2}+W_{11}^{(3)}(0) z \bar{z}+W_{02}^{(3)}(0) \frac{\bar{z}^{2}}{2}+\cdots
\end{aligned}
$$

we get

$$
\begin{aligned}
g(z, \bar{z}) & =\bar{q}^{*}(0) f_{0}(z, \bar{z})=f\left(0, x_{t}\right)=\bar{D} \tau_{k}\left(1, \bar{\alpha}^{*}, \bar{\beta}^{*}\right)\left(f_{1}^{(0)}, f_{2}^{(0)}, f_{3}^{(0)}\right)^{T} \\
& =\bar{D} \tau_{k}\left[f_{1}^{(0)}+\bar{\alpha}^{*} f_{2}^{(0)}+\bar{\beta}^{*} f_{3}^{(0)}\right]
\end{aligned}
$$




$$
\begin{aligned}
= & \bar{D} \tau_{k}\left[-\left(-l_{11} x_{1 t}^{2}(0)-l_{12} x_{1 t}(0) x_{2 t}(0)-l_{13} x_{1 t}(0) x_{3 t}(0)\right.\right. \\
& -l_{14} x_{2 t}(0) x_{3 t}(0)-l_{15} x_{1 t}^{2}(0) x_{2 t}(0)-l_{16} x_{1 t}^{3}(0)-l_{17} x_{1 t}^{3}(0) x_{2 t}(0) \\
& \left.-l_{18} x_{2 t}^{2}(0)-l_{19} x_{2 t}^{3}(0)-l_{110} x_{2 t}^{2}(0) x_{3 t}(0)+\cdots\right)+\bar{\alpha}^{*}\left(l_{21} x_{1 t}^{2}(0)\right. \\
& +l_{22} x_{1 t}(0) x_{2 t}(0)+l_{23} x_{1 t}^{3}(0)+l_{24} x_{1 t}^{3}(0) x_{2 t}(0)+l_{25} x_{1 t}^{2}(0) x_{2 t}(0) \\
& -l_{26} x_{1 t}(0) x_{3 t}(0)-l_{27} x_{2 t}(0) x_{3 t}(0)-l_{28} x_{1 t}^{2}(0) x_{3 t}(0) \\
& -l_{29} x_{2 t}^{2}(0) x_{3 t}(0)-l_{210} x_{2 t}^{3}(0)-l_{211} x_{2 t}^{3}(0) x_{3 t}(0) \\
& \left.-l_{212} x_{1 t}^{2}(0) x_{2 t}^{2}(0)-l_{213} x_{1 t}(0) x_{2 t}^{3}(0)+\cdots\right)+\bar{\beta}^{*}\left(l_{31} x_{3 t}^{2}(0)\right. \\
& -l_{32} x_{1 t}^{2}(0)-l_{33} x_{2 t}^{2}(0)-l_{34} x_{1 t}(0) x_{2 t}(0)-l_{35} x_{1 t}(0) x_{3 t}(0) \\
& -l_{36} x_{2 t}(0) x_{3 t}(0)-l_{37} x_{1 t}^{3}(0)-l_{38} x_{2 t}^{3}(0)-l_{39} x_{1 t}(0) x_{2 t}(0) x_{3 t}(0) \\
& -l_{310} x_{1 t}^{2}(0) x_{2 t}(0)-l_{311} x_{2 t}^{2}(0) x_{1 t}(0)-l_{312} x_{1 t}(0) x_{3 t}^{2}(0) \\
& \left.\left.-l_{313} x_{2 t}(0) x_{3 t}^{2}(0)-l_{314} x_{1 t}^{2}(0) x_{3 t}(0)-l_{315} x^{2}(0) x_{3 t}(0)+\cdots\right)\right]
\end{aligned}
$$

where

$$
\begin{aligned}
g_{20}= & 2 \bar{D} \tau_{k}\left[\left(-l_{11}+l_{12} \alpha+l_{13} \beta+l_{14} \alpha \beta+l_{18} \alpha^{2}\right)+\bar{\alpha}^{*}\left(l_{21}+l_{22} \alpha\right.\right. \\
& \left.-l_{26} \beta-l_{27} \alpha \beta+\bar{\beta}^{*}\left(l_{31} \beta^{2}-l_{32}-l_{33} \alpha^{2}-l_{34} \alpha-l_{35} \beta-l_{36} \alpha \beta\right)\right], \\
g_{11}= & 2 \bar{D} \tau_{k}\left[\left(-2 l_{11}+l_{12}(\alpha+\bar{\alpha})+l_{13}(\beta+\bar{\beta})+l_{14}(\alpha \bar{\beta}+\bar{\alpha} \beta)+2 l_{18}(\alpha \bar{\alpha})\right)\right. \\
& +\bar{\alpha}^{*}\left(2 l_{21}+l_{22}(\alpha+\bar{\alpha})-l_{26}(\beta+\bar{\beta})-l_{27}(\alpha \bar{\beta}+\bar{\alpha} \beta)\right)+\bar{\beta}^{*}\left(2 l_{31} \beta \bar{\beta}\right. \\
& \left.\left.-2 l_{32}-2 l_{33} \alpha \bar{\alpha}-l_{34}(\alpha+\bar{\alpha})-l_{35}(\beta+\bar{\beta})-l_{36}(\alpha \bar{\beta}+\bar{\alpha} \beta)\right)\right] \\
g_{02}= & 2 \bar{D} \tau_{k}\left[\left(-l_{11}+l_{12} \bar{\alpha}+l_{13} \bar{\beta}+l_{14} \bar{\alpha} \bar{\beta}+l_{18} \bar{\alpha}^{2}\right)\right. \\
& +\bar{\alpha}^{*}\left(2 l_{21}+l_{22} \bar{\alpha}-l_{26} \bar{\beta}-l_{27} \bar{\alpha} \bar{\beta}\right) \\
& \left.+\bar{\beta}^{*}\left(l_{31} \bar{\beta}^{2}-l_{32}-l_{33} \bar{\alpha}^{2}-l_{34} \bar{\alpha}-l_{35} \bar{\beta}-l_{36} \bar{\alpha} \bar{\beta}\right)\right] \\
g_{21}= & 2 \bar{D} \tau_{k}\left\{\left[\left(l_{15}(\alpha+\bar{\alpha})+l_{16}+l_{19} \alpha^{2} \bar{\alpha}\right)-l_{11}\left(2 W_{11}^{(1)}(0)+4 W_{20}^{(1)}(0)\right)\right.\right. \\
& +l_{12}\left(W_{11}^{(2)}(0)+2 W_{20}^{(2)}(0)+\alpha W_{11}^{(1)}(0)+2 \bar{\alpha} W_{20}^{(1)}(0)\right)+l_{13}\left(W_{11}^{(3)}(0)\right. \\
& \left.\left.+2 W_{20}^{(3)}(0)+\beta W_{11}^{(1)}(0)+2 \bar{\beta} W_{20}^{(1)}(0)\right)+l_{18}\left(2 \alpha W_{11}^{(2)}(0)+4 \bar{\alpha} W_{20}^{(2)}(0)\right)\right] \\
& +\alpha^{*}\left[l_{23}+l_{25}(\alpha+\bar{\alpha})-l_{28}(\beta+\bar{\beta})-l_{29}\left(\bar{\beta} \alpha^{2}+\bar{\alpha} \alpha \beta-l_{210} \beta^{2} \bar{\beta}\right)\right. \\
& +l_{21}\left(2 W_{11}^{(1)}(0)+4 W_{20}^{(1)}(0)\right)+l_{22}\left(W_{11}^{(2)}(0)+2 W_{20}^{(2)}(0)+\alpha W_{11}^{(1)}(0)\right. \\
& \left.+2 \bar{\alpha} W_{20}^{(1)}(0)\right)-l_{26}\left(W_{11}^{(3)}(0)+2 W_{20}^{(3)}(0)+\beta W_{11}^{(1)}(0)+2 \bar{\beta} W_{20}^{(1)}(0)\right) \\
& \left.-l_{27}\left(\alpha W_{11}^{(3)}(0)+2 \bar{\alpha} W_{20}^{(3)}(0)+\beta W_{11}^{(2)}(0)+2 \bar{\beta} W_{11}^{(2)}(0)\right)\right] \\
& +\beta^{*}\left[-l_{37}-l_{38} \alpha^{2} \bar{\alpha}-l_{39}(\alpha \bar{\beta} \bar{\alpha} \beta+\alpha \beta)-l_{310}(\beta+\bar{\beta})\right. \\
& -l_{311}\left(\alpha^{2}+2 \alpha \bar{\alpha}\right)-l_{312}\left(\beta^{2}+2 \beta \bar{\beta}\right)-l_{313}\left(\beta^{2} \bar{\alpha}+2 \beta \bar{\beta} \alpha\right) \\
& -l_{314}(\bar{\beta}+2 \beta)-l_{315}\left(\alpha^{2} \bar{\beta}+2 \alpha \bar{\alpha} \beta\right)+l_{31}\left(\beta W_{11}^{(3)}(0)+2 \bar{\beta} W_{20}^{(3)}(0)\right) \\
& -l_{32}\left(W_{11}^{(1)}(0)+2 W_{20}^{(1)}(0)\right)+l_{33}\left(\alpha W_{11}^{(2)}(0)+2 \bar{\alpha} W_{20}^{(2)}(0)\right) \\
& -l_{34}\left(W_{11}^{(2)}(0)+2 W_{20}^{(2)}(0)+\alpha W_{11}^{(1)}(0)+2 \bar{\alpha} W_{20}^{(1)}(0)\right) \\
&
\end{aligned}
$$




$$
\begin{aligned}
& -l_{35}\left(W_{11}^{(3)}(0)+2 W_{20}^{(3)}(0)+\beta W_{11}^{(1)}(0)+2 \bar{\beta} W_{20}^{(1)}(0)\right) \\
& \left.\left.-l_{36}\left(\alpha W_{11}^{(3)}(0)+2 \bar{\alpha} W_{20}^{(3)}(0)+\beta W_{11}^{(2)}(0)+2 \bar{\beta} W_{20}^{(2)}(0)\right)\right]\right\} .
\end{aligned}
$$

Since $\left.W_{11}(\theta), W_{20}(\theta)\right)$ in $g_{21}$ are unknown, we still need to compute them. By (3.6) and (3.8), we can derive

$$
\dot{W}=\left\{\begin{array}{ll}
A W-2 \operatorname{Re}\left\{\bar{q}^{*}(0) f_{0} q(\theta)\right\}, & \theta \in[-1,0) \\
A W-2 \operatorname{Re}\left\{\bar{q}^{*}(0) f_{0} q(\theta)\right\}+f_{0}, & \theta=0
\end{array} \triangleq A W+H(z, \bar{z}, \theta),\right.
$$

where

$$
H(z, \bar{z}, \theta)=H_{20}(\theta) \frac{z^{2}}{2}+H_{11}(\theta) z \bar{z}+H_{02}(\theta) \frac{\bar{z}^{2}}{2} \cdots .
$$

Notice that near the origin on the center manifold $C_{0}$, we have

$$
\dot{W}=W_{z} \dot{z}+W_{\bar{z}} \dot{\bar{z}}
$$

From (3.14), (3.15), and (3.16), comparing the coefficients, we get

$$
\begin{aligned}
& \left(A-2 i \omega_{0} \tau_{k} I\right) W_{20}(\theta)=-H_{20}(\theta), \\
& A W_{11}(\theta)=-H_{11}(\theta) .
\end{aligned}
$$

According to (3.14), we know that, for $\theta \in[-1,0)$,

$$
H(z, \bar{z}, \theta)=-\bar{q}^{*}(0) f_{0} q(\theta)-q^{*}(0) \bar{f}_{0} \bar{q}(\theta)=-g(z, \bar{z}) q(\theta)-\bar{g}(z, \bar{z}) \bar{q}(\theta) .
$$

Comparing the coefficients with (3.15), we obtain

$$
\begin{aligned}
& H_{20}(\theta)=-g_{20} q(\theta)-\bar{g}_{02} \bar{q}(\theta), \\
& H_{11}(\theta)=-g_{11} q(\theta)-\bar{g}_{11} \bar{q}(\theta) .
\end{aligned}
$$

By (3.17), (3.20), and the definition of $A$, it follows that

$$
\dot{W}_{20}(\theta)=2 i \omega_{0} \tau_{k} W_{20}(\theta)+g_{20} q(\theta)+\bar{g}_{02} \bar{q}(\theta) .
$$

Substituting $q(\theta)=(1, \alpha, \beta)^{T} e^{i \theta \omega_{0} \tau_{k}}$ into the equation, we can obtain the solution of it, which reads

$$
W_{20}(\theta)=\frac{i g_{20}}{\omega_{0} \tau_{k}} q(0) e^{i \theta \omega_{0} \tau_{k}}+\frac{i \bar{g}_{02}}{3 \omega_{0} \tau_{k}} \bar{q}(0) e^{-i \theta \omega_{0} \tau_{k}}+E_{1} e^{2 i \theta \omega_{0} \tau_{k}},
$$

where $E_{1}=\left(E_{1}^{(1)}, E_{1}^{(2)}, E_{1}^{(3)}\right)^{T} \in R^{3}$ is a constant vector.

Similarly, in view of (3.18), (3.21), and the definition of $A$, we can obtain

$$
\dot{W}_{11}(\theta)=g_{11} q(\theta)+\bar{g}_{11} \bar{q}(\theta) \text {, }
$$




$$
W_{11}(\theta)=-\frac{i g_{11}}{\omega_{0} \tau_{k}} q(0) e^{i \theta \omega_{0} \tau_{k}}+\frac{i \bar{g}_{11}}{\omega_{0} \tau_{k}} \bar{q}(0) e^{-i \theta \omega_{0} \tau_{k}}+E_{2},
$$

where $E_{2}=\left(E_{2}^{(1)}, E_{2}^{(2)}, E_{2}^{(3)}\right)^{T} \in R^{3}$ is a constant vector.

Next, we shall seek appropriate $E_{1}, E_{2}$, respectively. In the light of the definition of $A$, combined with (3.23) and (3.25), we have

$$
\dot{W}_{20}(\theta)=\int_{-1}^{0} d \eta(\theta) W_{20}(\theta)=2 i \omega_{0} \tau_{k} W_{20}(0)-H_{20}(0)
$$

and

$$
\dot{W}_{11}(\theta)=\int_{-1}^{0} d \eta(\theta) W_{11}(\theta)=-H_{11}(0)
$$

where $\eta(\theta)=\eta(0, \theta)$. From (3.17) and (3.18), we get

$$
\begin{aligned}
H_{20}(0)= & -g_{20} q(0)-\bar{g}_{02} \bar{q}(0) \\
& +2 \tau_{k}\left(\begin{array}{c}
-l_{11}+l_{12} \alpha+l_{13} \beta+l_{14} \alpha \beta+l_{18} \alpha^{2} \\
l_{21}+l_{22} \alpha-l_{26} \beta-l_{27} \alpha \beta \\
l_{31} \beta^{2}-l_{32}-l_{33} \alpha^{2}-l_{34} \alpha-l_{35} \beta-l_{36} \alpha \beta
\end{array}\right),
\end{aligned}
$$

and

$$
\begin{aligned}
H_{11}(0)= & -g_{11} q(0)-\bar{g}_{11} \bar{q}(0)+2 \tau_{k} \\
& \times\left(\begin{array}{c}
-2 l_{11}+l_{12}(\alpha+\bar{\alpha})+l_{13}(\beta+\bar{\beta})+l_{14}(\alpha \bar{\beta}+\bar{\alpha} \beta)+2 l_{18}(\alpha \bar{\alpha}) \\
2 l_{21}+l_{22}(\alpha+\bar{\alpha})-l_{26}(\beta+\bar{\beta})-l_{27}(\alpha \bar{\beta}+\bar{\alpha} \beta) \\
2 l_{31} \beta \bar{\beta}-2 l_{32}-2 l_{33} \alpha \bar{\alpha}-l_{34}(\alpha+\bar{\alpha})-l_{35}(\beta+\bar{\beta})-l_{36}(\alpha \bar{\beta}+\bar{\alpha} \beta)
\end{array}\right) .
\end{aligned}
$$

For $i \omega_{0} \tau_{k}$ is the eigenvalues of $A$ and $q(0)$ is the corresponding eigenvector, we obtain

$$
\begin{aligned}
& \left(i \omega_{0} \tau_{k} I-\int_{-1}^{0} e^{i \theta \omega_{0} \tau_{k}} d \eta(\theta)\right) q(0)=0, \\
& \left(-i \omega_{0} \tau_{k} I-\int_{-1}^{0} e^{-i \theta \omega_{0} \tau_{k}} d \eta(\theta)\right) \bar{q}(0)=0 .
\end{aligned}
$$

Substituting (3.23), (3.28) into (3.26), we obtain

$$
\begin{aligned}
& \left(2 i \omega_{0} \tau_{k} I-\int_{-1}^{0} e^{2 i \theta \omega_{0} \tau_{k}} d \eta(\theta)\right) E_{1} \\
& \quad=2 \tau_{k}\left(\begin{array}{c}
-l_{11}+l_{12} \alpha+l_{13} \beta+l_{14} \alpha \beta+l_{18} \alpha^{2} \\
l_{21}+l_{22} \alpha-l_{26} \beta-l_{27} \alpha \beta \\
l_{31} \beta^{2}-l_{32}-l_{33} \alpha^{2}-l_{34} \alpha-l_{35} \beta-l_{36} \alpha \beta
\end{array}\right) .
\end{aligned}
$$

That is,

$$
\left(\begin{array}{ccc}
2 i \omega_{0}-a_{11}+k_{1} e^{-i \omega_{0} \tau_{k}} & -a_{12} & -a_{13} \\
-a_{21} & 2 i \omega_{0}-a_{22}+k_{2} e^{-i \omega_{0} \tau_{k}} & -a_{23} \\
-a_{31} & -a_{32} & 2 i \omega_{0}-a_{33}+k_{3} e^{-i \omega_{0} \tau_{k}}
\end{array}\right)
$$




$$
\begin{aligned}
E_{1} & =\left(\begin{array}{ccc}
\varrho_{1} & -a_{12} & -a_{13} \\
-a_{21} & \varrho_{2} & -a_{23} \\
-a_{31} & -a_{32} & \varrho_{3}
\end{array}\right) E_{1} \\
& =2\left(\begin{array}{c}
-l_{11}+l_{12} \alpha+l_{13} \beta+l_{14} \alpha \beta+l_{18} \alpha^{2} \\
l_{21}+l_{22} \alpha-l_{26} \beta-l_{27} \alpha \beta \\
l_{31} \beta^{2}-l_{32}-l_{33} \alpha^{2}-l_{34} \alpha-l_{35} \beta-l_{36} \alpha \beta
\end{array}\right)=2\left(\begin{array}{l}
\aleph_{1} \\
\aleph_{2} \\
\aleph_{3}
\end{array}\right) .
\end{aligned}
$$

It follows that

$$
E_{1}^{(1)}=\frac{\Delta_{11}}{\Delta_{1}}, \quad E_{1}^{(2)}=\frac{\Delta_{12}}{\Delta_{1}}, \quad E_{1}^{(3)}=\frac{\Delta_{13}}{\Delta_{1}},
$$

where

$$
\begin{aligned}
& \Delta_{1}=\left|\begin{array}{ccc}
\varrho_{1} & -a_{12} & -a_{13} \\
-a_{21} & \varrho_{2} & -a_{23} \\
-a_{31} & -a_{32} & \varrho_{3}
\end{array}\right|, \\
& \Delta_{11}=2\left|\begin{array}{ccc}
\aleph_{1} & -a_{12} & -a_{13} \\
\aleph_{2} & \varrho_{2} & -a_{23} \\
\aleph_{3} & -a_{32} & \varrho_{3}
\end{array}\right|, \\
& \Delta_{12}=2\left|\begin{array}{ccc}
\varrho_{1} & \aleph_{1} & -a_{13} \\
-a_{21} & \aleph_{2} & -a_{23} \\
-a_{31} & \aleph_{3} & \varrho_{3}
\end{array}\right|, \\
& \Delta_{13}=2\left|\begin{array}{ccc}
\varrho_{1} & -a_{12} & \aleph_{1} \\
-a_{21} & \varrho_{2} & \aleph_{2} \\
-a_{31} & -a_{32} & \aleph_{3}
\end{array}\right| .
\end{aligned}
$$

Similarly, substituting (3.24), (3.29) into (3.27), we have

$$
\begin{aligned}
& \left(\begin{array}{ccc}
-a_{11}+k_{1} & -a_{12} & -a_{13} \\
-a_{21} & -a_{22}+k_{2} & -a_{23} \\
-a_{31} & -a_{32} & -a_{33}+k_{3}
\end{array}\right) E_{2} \\
& =2\left(\begin{array}{c}
-2 l_{11}+l_{12}(\alpha+\bar{\alpha})+l_{13}(\beta+\bar{\beta})+l_{14}(\alpha \bar{\beta}+\bar{\alpha} \beta)+2 l_{18}(\alpha \bar{\alpha}) \\
2 l_{21}+l_{22}(\alpha+\bar{\alpha})-l_{26}(\beta+\bar{\beta})-l_{27}(\alpha \bar{\beta}+\bar{\alpha} \beta) \\
2 l_{31} \beta \bar{\beta}-2 l_{32}-2 l_{33} \alpha \bar{\alpha}-l_{34}(\alpha+\bar{\alpha})-l_{35}(\beta+\bar{\beta})-l_{36}(\alpha \bar{\beta}+\bar{\alpha} \beta)
\end{array}\right) \\
& =2\left(\begin{array}{c}
\hbar_{1} \\
\hbar_{2} \\
\hbar_{3}
\end{array}\right) .
\end{aligned}
$$

It follows that

$$
E_{2}^{(1)}=\frac{\Delta_{21}}{\Delta_{2}}, \quad E_{2}^{(2)}=\frac{\Delta_{22}}{\Delta_{2}}, \quad E_{2}^{(3)}=\frac{\Delta_{23}}{\Delta_{2}},
$$


where

$$
\begin{aligned}
& \Delta_{2}=\left|\begin{array}{ccc}
-a_{11}+k_{1} & -a_{12} & -a_{13} \\
-a_{21} & -a_{22}+k_{2} & -a_{23} \\
-a_{31} & -a_{32} & -a_{33}+k_{3}
\end{array}\right|, \\
& \Delta_{21}=2\left|\begin{array}{ccc}
\hbar_{1} & -a_{12} & -a_{13} \\
\hbar_{2} & -a_{22}+k_{2} & -a_{23} \\
\hbar_{3} & -a_{32} & -a_{33}+k_{3}
\end{array}\right|, \\
& \Delta_{22}=2\left|\begin{array}{ccc}
-a_{11}+k_{1} & \hbar_{1} & -a_{13} \\
-a_{21} & \hbar_{2} & -a_{23} \\
-a_{31} & \hbar_{3} & -a_{33}+k_{3}
\end{array}\right|, \\
& \Delta_{23}=2\left|\begin{array}{ccc}
-a_{11}+k_{1} & -a_{12} & \hbar_{1} \\
-a_{21} & -a_{22}+k_{2} & \hbar_{2} \\
-a_{31} & -a_{32} & \hbar_{3}
\end{array}\right| .
\end{aligned}
$$

Consequently, we can determine $W_{20}(0)$ and $W_{11}(0)$ from (3.24), (3.26). Furthermore, based on the above calculation, we can compute $g_{21}$. Therefore, all $g_{i j}$ are determined by the parameters and delay in (3.1). After that, we can easily compute the following values:

$$
\begin{aligned}
& C_{1}(0)=\frac{i}{2 \omega_{0} \tau_{k}}\left(g_{11} g_{20}-2\left|g_{11}\right|^{2}-\frac{1}{3}\left|g_{02}\right|^{2}\right)+\frac{g_{21}}{2}, \\
& \mu_{2}=-\frac{\operatorname{Re}\left\{C_{1}(0)\right\}}{\operatorname{Re}\left\{\lambda^{\prime}\left(\tau_{k}\right)\right\}} \\
& \beta_{2}=2 \operatorname{Re}\left\{C_{1}(0)\right\}, \\
& T_{2}=-\frac{\operatorname{Im}\left\{C_{1}(0)\right\}+\mu_{2} \operatorname{Im}\left\{\lambda^{\prime}\left(\tau_{k}\right)\right\}}{\omega_{0} \tau_{k}} .
\end{aligned}
$$

Thus, we have the main results of this section.

Theorem 2 For (3.32), we have

(i) The sign of $\mu_{2}$ determines the direction of the Hopf bifurcation, if $\mu_{2}>0\left(\mu_{2}<0\right)$, then the Hopf bifurcation is supercritical (subcritical) and the bifurcating period solutions exist for $\tau>\tau_{k}\left(\tau<\tau_{k}\right)$;

(ii) The sign of $\beta_{2}$ determines the stability of the bifurcating period solutions, if $\beta_{2}<0\left(\beta_{2}>0\right)$, then the bifurcation period solutions are orbital stable (unstable);

(iii) The sign of $T_{2}$ determines the period of the bifurcating period solutions, if $T_{2}>0\left(T_{2}<0\right)$, then the period increases (decreases).

\section{Application to control of chaos}

In this section, extensive numerical simulations are carried out to verify the analytical results of the tri-trophic food web system for a Leslie-Gower type generalist predator model. Consider the following set of data for system (1.2):

$$
\begin{array}{ll}
w_{1}=1.4, & w_{2}=5, \quad w_{3}=8, \quad w_{4}=1, \quad w_{5}=0.16, \quad w_{6}=0.1, \\
w_{7}=0.1, \quad w_{8}=0.5, \quad w_{9}=8, & w_{10}=8 .
\end{array}
$$


Now, we give numerical simulations to confirm our theoretical analysis. According to the above related parameters, we can obtain

$$
\left\{\begin{array}{l}
\frac{d x}{d t}=x\left[1-x-\frac{y}{1+1.4 x}-\frac{z}{1+5 x+8 y}\right]+k_{1}[x-x(t-\tau)] \\
\frac{d y}{d t}=y\left[-0.16+\frac{x}{1+1.4 x}-\frac{0.1 z}{1+5 x+8 y}\right]+k_{2}[y-y(t-\tau)] \\
\left.\frac{d z}{d t}=0.1 z^{2}-\frac{0.5 z^{2}}{1+8 x+8 y}\right)+k_{3}[z-z(t-\tau)]
\end{array}\right.
$$

which has a positive equilibrium $E_{+}=\left(\frac{473}{1530}, \frac{146}{765}, \frac{1483}{653}\right)$. The linearized system of system (4.1) near $E_{+}$takes the form

$$
\dot{u}(t)=\left(\begin{array}{ccc}
-0.0573 & 0.1229 & -0.0759 \\
0.1060 & 0.0209 & -0.0047 \\
0.8252 & 0.8252 & 0
\end{array}\right) u(t)+\left(\begin{array}{ccc}
-k_{1} & 0 & 0 \\
0 & -k_{2} & 0 \\
0 & 0 & -k_{3}
\end{array}\right) u(t-\tau)
$$

where $u(t)=(x(t), y(t), z(t))^{T}$. The characteristic equation of system (4.2) is

$$
\begin{aligned}
& \left(\lambda^{3}+a_{2}^{*} \lambda^{2}+a_{1}^{*} \lambda+a_{0}\right) e^{\lambda \tau}+b_{2}^{*} \lambda^{2}+b_{1}^{*} \lambda+b_{0}^{*}+\left(c_{1}^{*} \lambda+c_{0}^{*}\right) e^{-\lambda \tau}+d_{0}^{*} e^{-2 \lambda \tau}=0, \\
& a_{2}^{*}=0.0364, \quad a_{1}^{*}=0.0523, \quad a_{0}^{*}=0.006, \quad b_{2}^{*}=k_{1}+k_{2}+k_{3}, \\
& b_{1}^{*}=-0.0209 k_{1}+0.0573 k_{2}+0.0364 k_{3}, \quad b_{0}^{*}=0.0039 k_{1}+0.0626 k_{2}-0.0142 k_{3}, \\
& c_{1}^{*}=k_{1} k_{2}+k_{1} k_{3}+k_{2} k_{3}, \quad c_{0}^{*}=-0.0209 k_{1} k_{3}+0.0573 k_{2} k_{3}, \quad d_{0}^{*}=k_{1} k_{2} k_{3} .
\end{aligned}
$$

When $\tau=0$ or $k_{1}=k_{2}=k_{3}=0$, then (4.3) becomes

$$
\lambda^{3}+0.0363 \lambda^{2}+0.0523 \lambda+0.006=0 .
$$

It is clear that the positive equilibrium $E_{+}$is unstable. System (4.1) has a chaotic attractor (see Fig. 1).

When $\tau \neq 0$ or $k_{1} \neq 0, k_{2} \neq 0, k_{3} \neq 0$, then Eq. (4.3) becomes

$$
\begin{aligned}
& \left(\lambda^{3}+0.0364 \lambda^{2}+0.0523 \lambda+0.006\right) e^{\lambda \tau}+\left(k_{1}+k_{2}+k_{3}\right) \lambda^{2} \\
& \quad+\left(0.0364 k_{3}+0.0573 k_{2}-0.0209 k_{1}\right) \lambda+\left(0.0039 k_{1}+0.0626 k_{2}-0.0142 k_{3}\right) \\
& +\left(\left(k_{1} k_{2}+k_{1} k_{3}+k_{2} k_{3}\right) \lambda+\left(0.0573 k_{2} k_{3}-0.0209 k_{1} k_{3}\right)\right) e^{-\lambda \tau} \\
& \quad+\left(k_{1} k_{2} k_{3}\right) e^{-2 \lambda \tau}=0 .
\end{aligned}
$$

Set $k_{1}=k_{2}=k_{3}=-0.02$, we can obtain $\omega_{0} \approx 0.20298, \tau_{0} \approx 7.739$, then $\operatorname{Re} C_{1}(0)=1.5562$. When $\tau$ passes through the critical values $\tau_{k}$, the positive equilibrium $E_{+}$loses its stability and a Hopf bifurcation occurs. Numerical simulation shows the bifurcation diagrams of system (4.1) (see Fig. 2). Figure 3 shows the positive equilibrium $E_{+}$is still chaotic when $\tau=4$. And the positive equilibrium is asymptotically stable (see Fig. 4). Notice that $\mu_{2}<0$ and $\beta_{2}>0$, the Hopf bifurcation is subcritical and the bifurcating periodic solutions from the equilibrium $E_{+}$are unstable (see Fig. 5).

From the numerical simulation, it can be seen that when the delay feedback is incorporated in a Leslie-Gower type generalist predator model in a tri-trophic food web system, 


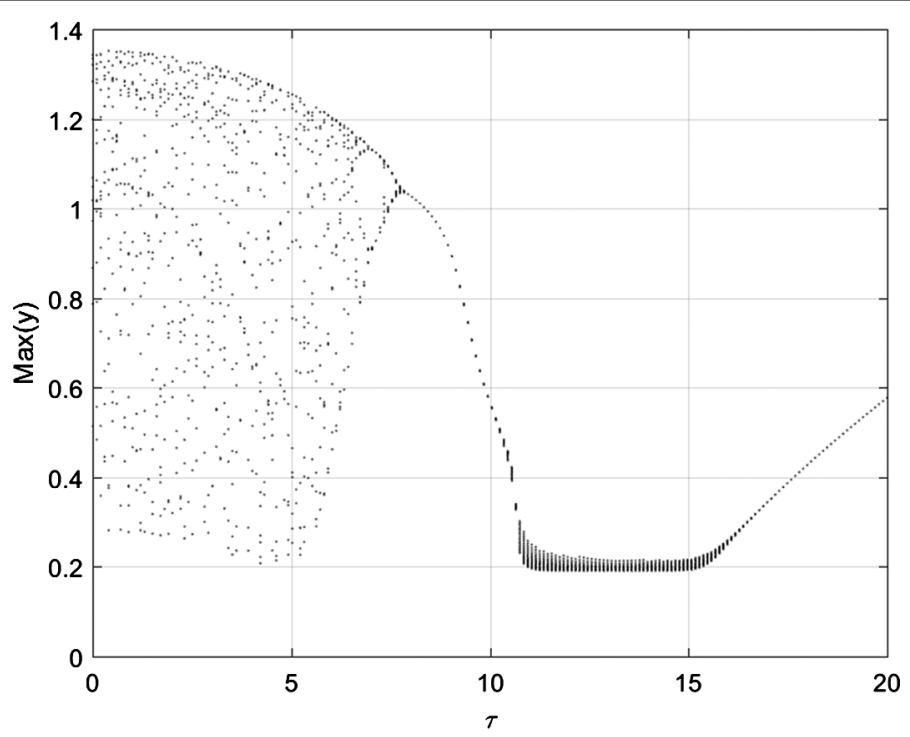

Figure 2 The bifurcation diagram of system (4.1), where $w_{1}=1.4, w_{2}=5, w_{3}=8, w_{4}=1, w_{5}=0.16, w_{6}=0.1$, $w_{7}=0.1, w_{8}=0.5, w_{9}=8, w_{10}=8, k_{1}=-0.02, k_{2}=-0.02, k_{3}=-0.02$
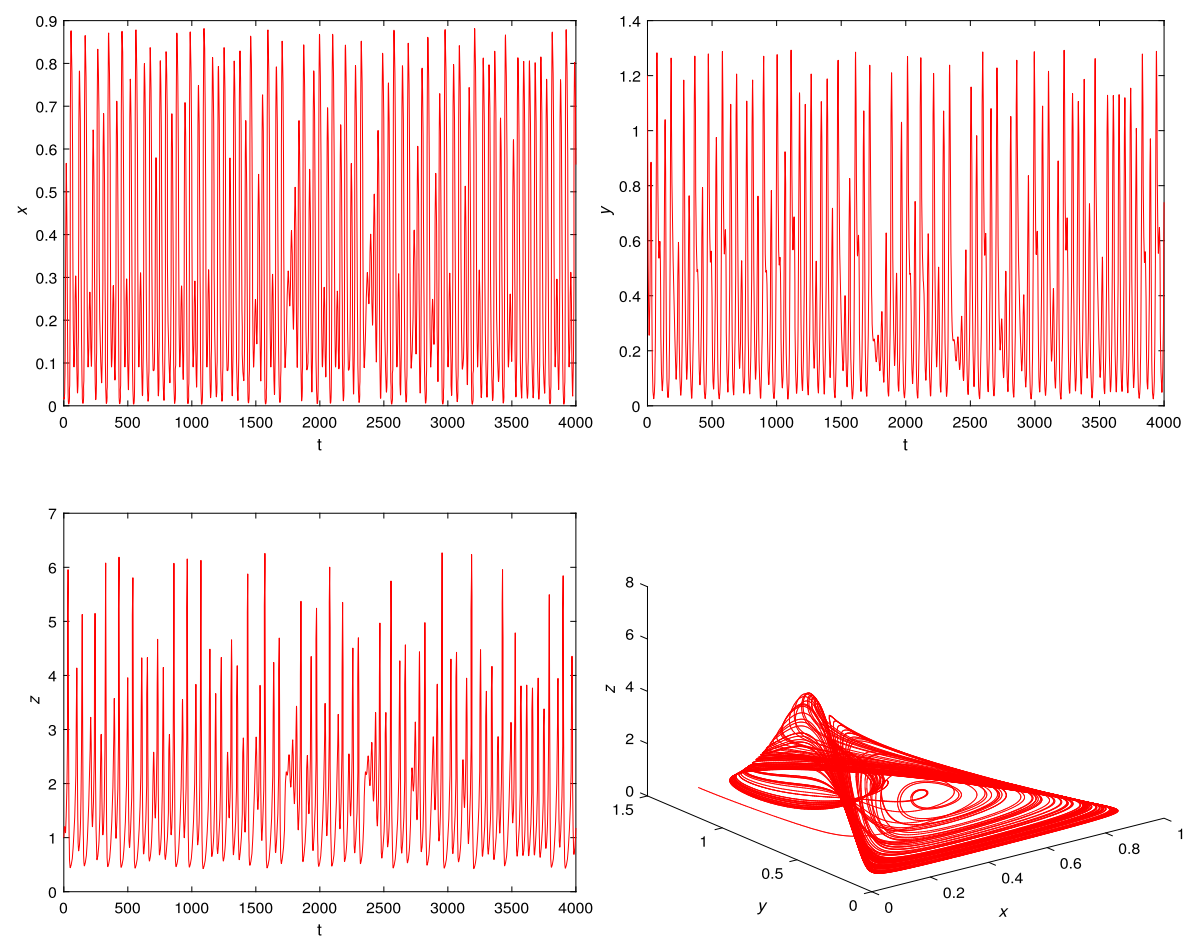

Figure 3 The trajectories and graphs of system (4.1) with $\tau=4$. The positive equilibrium $E^{+}$still has a chaotic attractor

rich and colorful dynamical behaviors can occur by adjusting the appropriate feedback strength $k$ and time delay $\tau$. Especially when the time delay reaches a certain value, the chaotic attractor vanishes. In this sense, delayed feedback successfully implements chaos control. 


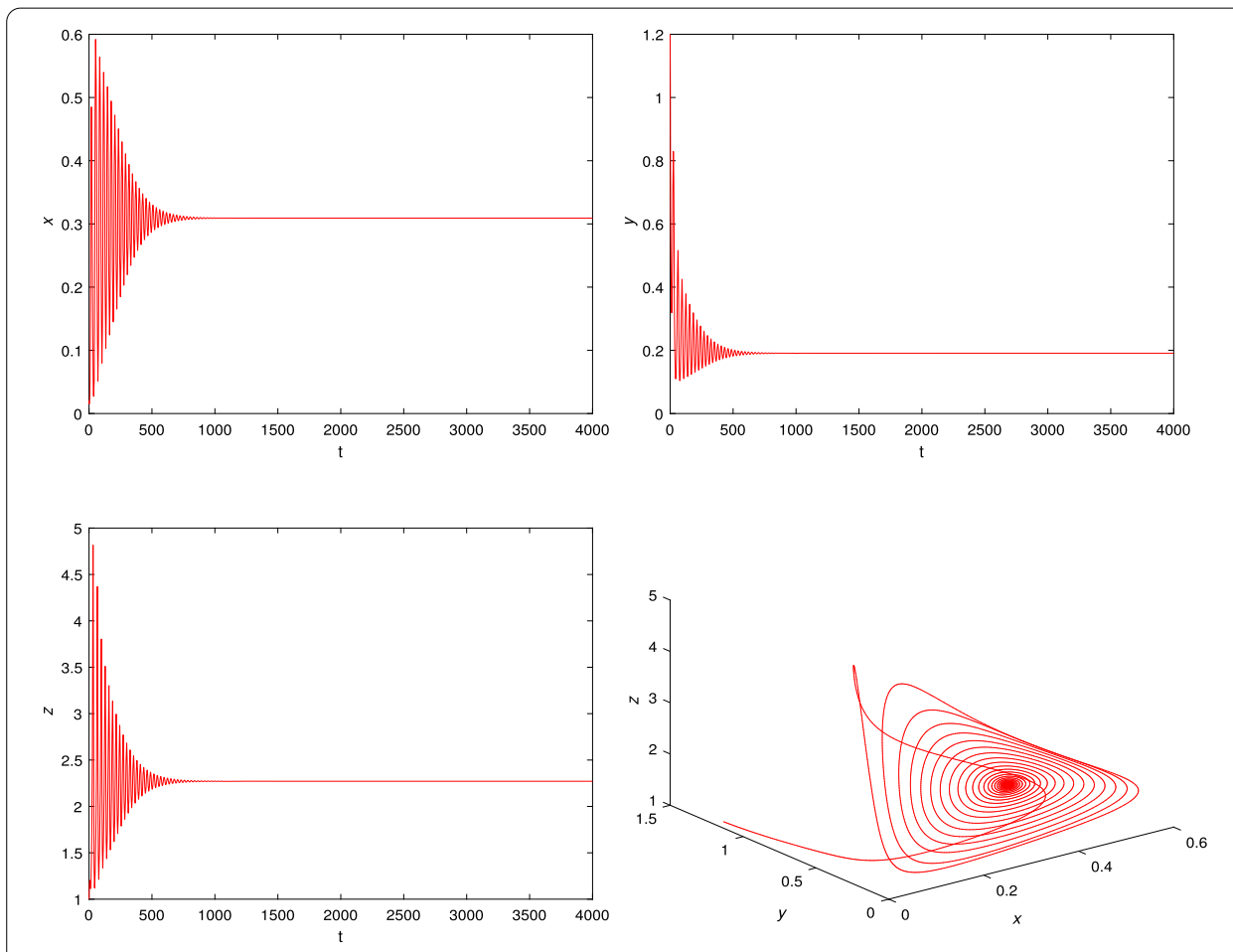

Figure 4 The trajectories and graphs of system (4.1) with $\tau=12$. The positive equilibrium $E^{+}$is asymptotically stable
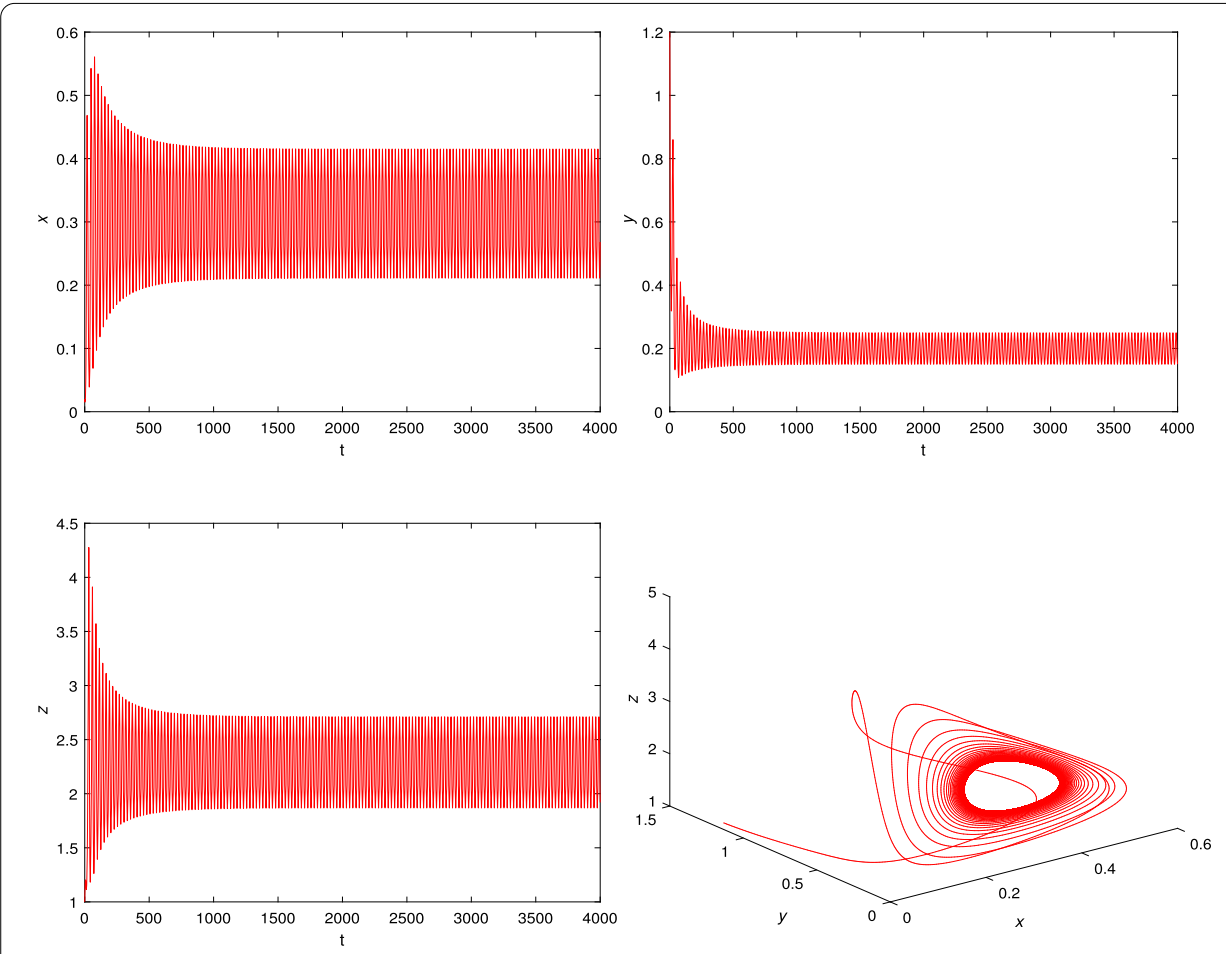

Figure 5 The trajectories and graphs of system (4.1) with $\tau=16$. The positive equilibrium $E^{+}$is unstable, and a stable periodic solution from $E^{+}$ 


\section{Conclusions}

This paper provides stability and bifurcation analysis in a tri-trophic food web model with Leslie-Gower type generalist predator with delay feedback. Some conditions are given to ensure the existence of Hopf bifurcation occurring at the positive equilibrium by investigating the associated characteristic equation. The properties of Hopf bifurcations, such as the direction and stability of periodic solutions, are investigated based on the normal form theory and center manifold theorem. With the help of some numerical simulation, we show the exact value where chaos appears or vanishes. Our results show that if we choose some appropriate parameters, the oscillation can be controlled to a stable equilibrium or a stable periodic orbit. That is to say, we can achieve the three species coexistence by adjusting the capture (or release) level. Moreover, the control method used in this paper can be applicable to other chaotic systems.

\footnotetext{
Acknowledgements

The authors would like to express their appreciation to the editors and the anonymous referees for their many valuable suggestions and for carefully correcting the preliminary version of the manuscript.
}

\section{Funding}

This work was supported by the National Natural Science Foundation of China (Grant No. 61761002), the First-Class Disciplines Foundation of Ningxia (Grant No. NXYLXK2017B09).

Competing interests

The authors declare that they have no competing interests.

Authors' contributions

The authors contributed equally in this article. They read and approved the final manuscript.

\section{Publisher's Note}

Springer Nature remains neutral with regard to jurisdictional claims in published maps and institutional affiliations.

Received: 24 August 2018 Accepted: 15 July 2019 Published online: 02 August 2019

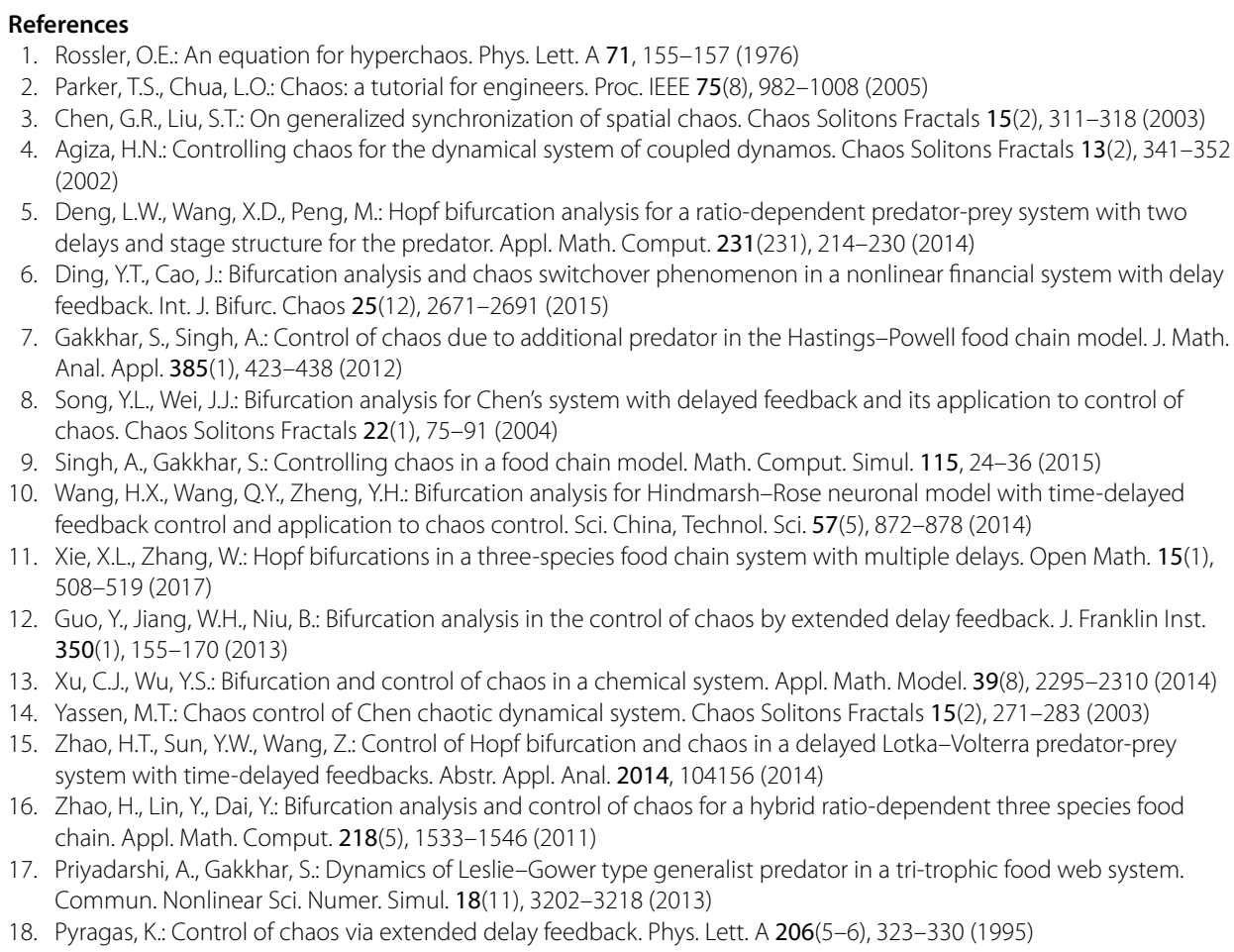


19. Jiang, Z.C., Ma, W.B.: Delayed feedback control and bifurcation analysis in a chaotic chemostat system. Int. J. Bifurc Chaos 25(6), 1550087 (2015)

20. Meng, X.Y., Huo, H.F., Zhang, X.B., Xiang, H.: Stability and Hopf bifurcation in a three-species system with feedback delays. Nonlinear Dyn. 64(4), 349-364 (2011)

21. Pal, P.J., Mandal, P.K.: Bifurcation analysis of a modified Leslie-Gower predator-prey model with Beddington DeAngelis functional response and strong Allee effect. Math. Comput. Simul. 97(1), 123-146 (2014)

22. Ruan, S.G., Wei, J.J.: On the zeros of a third degree exponential polynomial with applications to a delayed model for the control of testosterone secretion. IMA J. Math. Appl. Med. Biol. 18(1), 41-52 (2001)

23. Lancaster, P., Tismenetsky, M.: The Theory of Matrices: With Applications. Elsevier, Amsterdam (1985)

24. Cookea, K.L., Grossman, Z.: Discrete delay, distributed delay and stability switches. J. Math. Anal. Appl. 86(2), 592-627 (1982)

25. Kuang, Y.: Delay Differential Equations: With Applications in Population Dynamics. Academic Press, Pittsburgh (1993)

26. Hale, J.K.: Theory of Functional Differential Equations. Springer, Berlin (1977)

27. Hassard, B.D., Kazarinoff, N.D., Wan, Y.H.: Theory and Applications of Hopf Bifurcation. Cambridge University Press, Cambridge (1981)

28. Ruan, S.G., Wei, J.J.: On the zeros of transcendental functions with applications to stability of delay differential equations with two delays. Dyn. Contin. Discrete Impuls. Syst., Ser. A Math. Anal. 10(6), 863-874 (2003)

\section{Submit your manuscript to a SpringerOpen ${ }^{\circ}$ journal and benefit from:}

- Convenient online submission

- Rigorous peer review

- Open access: articles freely available online

- High visibility within the field

- Retaining the copyright to your article

Submit your next manuscript at $\boldsymbol{\nabla}$ springeropen.com 